\title{
DICRETE HÖLDER ESTIMATES FOR A CERTAIN KIND OF PARAMETRIX. II
}

\author{
A.I. PARFENOV
}

\begin{abstract}
In the first paper of this series we have introduced a certain parametrix and the associated potential. The parametrix corresponds to a uniformly elliptic second order differential operator with locally Hölder continuous coefficients in the half-space. Here we show that the potential is an approximate left inverse of the differential operator modulo hyperplane integrals, with the error estimated in terms of the local Hölder norms. As a corollary, we calculate approximately the potential whose density and differential operator originate from the straightening of a special Lipschitz domain. This corollary is aimed for the future derivation of approximate formulae for harmonic functions.
\end{abstract}

Keywords: cubic discretization, Lipschitz domain, local Hölder norms, parametrix, potential, straightening.

Mathematics Subject Classification: 35A17

\section{INTRODUCTION}

Let $\mathcal{A}_{\lambda}^{\mu}$ be the family of all second order uniformly elliptic operators in the upper half-space $\mathbb{R}_{+}^{n}(n \geqslant 2)$ with an ellipticity constant $\lambda \geqslant 1$ and locally $\mu$-Hölder coefficients, $0<\mu<1$. In work [1] a $Z$-parametrix $E(A ; x, y)$ (shortly: parametrix) was proposed for an operator $A \in \mathcal{A}_{\lambda}^{\mu}$ and for the corresponding potential

$$
\Phi_{f}(x)=\int_{y_{n}>0} E(A ; x, y) f(y) d y, \quad x \in \mathbb{R}_{+}^{n},
$$

estimates for local Hölder norms $\left\|D^{\alpha} \Phi_{f}\right\|_{I}(|\alpha| \leqslant 2)$ and $\|R f\|_{I}$ were established in terms of the same norms $\|f\|_{J}$, where $f \mapsto R f=f-A \Phi_{f}$ is the error operator.

The parametrix $E(A ; x, y)$ and the potential $\Phi_{f}$ were introduced in order to study a special harmonic function. Let $\Omega$ be the overgraph of a Lipschitz function $\omega: \mathbb{R}^{n-1} \rightarrow \mathbb{R}$. Lemma 3.7 in [2] and the properties of the Kelvin transform imply the existence and the uniqueness up to a positive multiplicative constant of a function $U$ with the following properties:

$$
U \in C^{\infty}(\Omega) \cap C(\bar{\Omega}), \quad \Delta U=0 \text { and } U>0 \text { in domain } \Omega,\left.\quad U\right|_{\partial \Omega}=0 .
$$

Up to the equivalence, the function $U$ determines the behavior of arbitrary positive harmonic functions vanishing continuously on a part of the boundary of a Lipschitz domain. Indeed, roughly speaking, each two such functions are comparable by the boundary Harnack principle. As an example see [3, Thm. 5.1].

Let us outline the plan of studying the function $U$. Denoting

$$
u=(U \circ g) \varphi
$$

A.I. Parfenov, Dicrete Hölder estimates for a Certain kind of Parametrix. II.

(C) PARfEnov A.I. 2017.

Submitted March 15, 2016. 
for an appropriate straightening diffeomorphism $g: \mathbb{R}_{+}^{n} \rightarrow \Omega$ and a cut-off function $\varphi \in$ $C_{0}^{\infty}\left(\overline{\mathbb{R}_{+}^{n}}\right)$, by the Laplace equation $\Delta U=0$ we obtain the differential equation

$$
A u=L D_{n} u+L^{\prime}
$$

for some operator $A \in \mathcal{A}_{\lambda}^{\mu}$ and functions $L, L^{\prime} \in C^{\infty}\left(\mathbb{R}_{+}^{n}\right)$. Here $A$ and $L$ depend on $\Omega$ and $g$, but not on $U$ and $\varphi$. If the function $\omega$ is compactly supported and its Lipschitz constant is sufficiently small, the Neumann series

$$
Q=\sum_{k=0}^{\infty} R^{k}
$$

makes sense. The boundary condition $\left.u\right|_{\partial \mathbb{R}_{+}^{n}}=0$ and the boundedness of the support of the function $u$ are preconditions for the validity of the integral representation

$$
u=\Phi_{F}, \quad F=Q A u=Q\left(v L+L^{\prime}\right), \quad v=D_{n} u=D_{n} \Phi_{F} .
$$

For the function $\mathbf{x}_{n}^{-1}(x)=x_{n}^{-1}$ and a number $v_{0}$ we write

$$
v=\mathbf{x}_{n}^{-1} \Phi_{F}\{\underbrace{D_{n} \Phi_{L}-\mathbf{x}_{n}^{-1} \Phi_{L}+1}_{\Theta}\}+\underbrace{D_{n} \Phi_{F-v_{0} L}-\mathbf{x}_{n}^{-1} \Phi_{F-v_{0} L}}_{\Theta_{1}}+\{\underbrace{D_{n} \Phi_{L}-\mathbf{x}_{n}^{-1} \Phi_{L}}_{\Theta_{2}}\}\{\underbrace{v_{0}-\mathbf{x}_{n}^{-1} \Phi_{F}}_{\Theta_{3}}\} .
$$

It turns out that $\Theta \approx \mathbf{x}_{n} D_{n}(S \circ g)$, where

$$
S(x)=\lim _{r \rightarrow \infty}\left\{\ln r-\frac{\Gamma(n / 2)}{\pi^{n / 2}} \int_{y \in \mathbb{R}^{n} \backslash \Omega:|x-y|<r}|x-y|^{-n} d y\right\}, \quad x \in \Omega,
$$

and the approximate error is quadratic in approximation numbers $b_{I}$ expressing how close locally the surface $\partial \Omega$ is to a hyperplane. We can choose $v_{0}$ so that the term $\Theta_{1}$ is estimated quadratically in $b_{I}$, while the expressions $\Theta_{2}$ and $\Theta_{3}$ are estimated linearly. This implies that $\frac{D_{n} u}{u} \approx D_{n}(S \circ g)$ with a quadratic error. Generalizing the arguments and the definition of the function $S$ to the case of a not necessarily compactly supported function $\omega$ with an arbitrary Lipschitz constant, by means of rotations of the coordinate system we obtain the approximate formula

$$
\frac{\nabla U}{U} \approx \nabla S
$$

The integration of this formula gives rise to the exponential asymptotic formula (EAF)

$$
U \approx U_{0} \mathrm{e}^{S} .
$$

For known EAFs for conformal mappings, EAFs for solutions to elliptic systems and asymptotics for positive harmonic functions see works [4]-[8].

The present paper is devoted to realizing a part of the outlined plan, namely, to justifying, for error term in the formula $\Theta \approx \mathbf{x}_{n} D_{n}(S \circ g)$, an estimate quadratic in approximating numbers of the function $\omega$. The paper consists of the introduction and two sections. In Section 2 we find approximately the potential $\Phi_{A f}$. The main definitions are given in Subsections 2.1 and 2.2. In Subsection 2.3, the discrete Hölder estimates from [1] for the functions $D^{\alpha} \Phi_{f}$ and $R f$ are completed by an estimate for the expression $D_{n} \Phi_{f}-\mathbf{x}_{n}^{-1} \Phi_{f}$, which is more precise than the independent estimates for the functions $\Phi_{f}$ and $D_{n} \Phi_{f}$. In Subsection 2.4, the derivatives $D^{\alpha} \Phi_{A f}$ and the expression $D_{n} \Phi_{A f}-\mathbf{x}_{n}^{-1} \Phi_{A f}$ are found up to the errors majorized by local Hölder seminorms $|A|_{J}$ of the coefficients of the operator $A$ and by the norms $\left\|D^{2} f\right\|_{J}$.

In Section 3, to a pair $(\omega, \theta)$, where $\theta \geqslant\|\omega\|_{\text {Lip }}$, we associate the standard set

$$
\left(\left\{\gamma_{K}\right\}, w, W, g, \mathfrak{g}, \mathfrak{G}, A, \lambda, L\right)
$$

relating to a straightening of the domain $\Omega$, after that the formula $\Theta \approx \mathbf{x}_{n} D_{n}(S \circ g)$ and its analogue for the derivatives $D_{i j} \Phi_{L}$ are established by a reduction to Subsection 2.4. We observe 
that the formula for the derivatives $D_{i j} \Phi_{L}$ can be used while obtaining an analogue of formula (1) for the derivatives $D_{i j} U$.

Convention. The letter $c$ (with a possible subscript or superscript) stands for various positive constants and always equipped by the brackets with all numerical parameters, on which these constants depend. For $t>0$ and a cube or a ball $X \subset \mathbb{R}^{d}$ centered at $\mathfrak{c}_{X}$ and of the edge or radius of an arbitrary length we let

$$
t X=\left\{\mathfrak{c}_{X}+t\left(\xi-\mathfrak{c}_{X}\right): \xi \in X\right\} .
$$

If $\xi \in \mathbb{R}^{d}$, then $|\xi|_{\infty}=\max _{i}\left|\xi_{i}\right|$ and (if $\xi$ is not a multi-index) $|\xi|^{2}=\sum_{i}\left|\xi_{i}\right|^{2}$. For multi-indices $\alpha \in \mathbb{N}_{0}^{d}$, by $D^{\alpha} f$ we denote partial derivatives of a real function $f$, at that, $D_{i} f \equiv D^{e_{i}} f$ and $D_{i j} f \equiv D^{e_{i}+e_{j}} f$, where $\left\{e_{i}\right\}_{1}^{d}$ is the canonical basis in $\mathbb{R}^{d}$. For a semi-norm $p$ and a number $q \in \mathbb{N}_{0}$ we let

$$
p\left(D^{q} f\right)=\max _{|\alpha|=q} p\left(D^{\alpha} f\right) .
$$

For instance, $|D f|=\max _{1 \leqslant i \leqslant d}\left|D_{i} f\right|=|\nabla f|_{\infty}$, where $\nabla f$ is the gradient of the function $f$. By $\bar{X}$ and $X^{\circ}$ we denote the closure and the interior of a set $X \subset \mathbb{R}^{d}$.

\section{Approximate calculations with potential $\Phi_{A f}$}

2.1. Basic information on a dyadic family. Given an integer $n \geqslant 2$, we introduce a dyadic family $\mathcal{D}$ in $\mathbb{R}^{n-1}$ :

$$
\mathcal{D}=\bigcup_{k \in \mathbb{Z}} \mathcal{D}_{k}, \quad \mathcal{D}_{k}=\left\{I: I=\left[0,2^{k}\right)^{n-1}+2^{k} a \text { for some } a \in \mathbb{Z}^{n-1}\right\} .
$$

For the sets $I_{i} \subset \mathbb{R}^{n-1}$ with a bounded non-empty union we let

$$
\left[I_{1}, I_{2}\right]=\sup _{\xi, \eta \in I_{1} \cup I_{2}}|\xi-\eta|_{\infty} .
$$

We denote $l_{I}=[I, \varnothing]$ as $I \in \mathcal{D}$ (the side-length). For $\alpha, \beta \in \mathbb{R}$ we let

$$
\Gamma_{I J}^{(\alpha, \beta)}=l_{I}^{\alpha} l_{J}^{\beta}[I, J]^{-\alpha-\beta}, \quad I, J \in \mathcal{D} .
$$

The following statements is Theorem 2(a) proved in [9]. Hereinafter, unless otherwise said, the summation are taken over the set $\mathcal{D}$.

Lemma 1. If $\alpha>0$ and $\beta>n-1$, then

$$
\sum_{J} \Gamma_{I J}^{(\alpha, \beta)} \leqslant c(n, \alpha, \beta), \quad I \in \mathcal{D}
$$

For $I, J \in \mathcal{D}$ we say $I \odot J$ if $l_{I}=l_{J}$ and $\bar{I} \cap \bar{J} \neq \varnothing$. By $\left\{I^{J}, J^{I}\right\}$ we denote a pair of cubes $\left\{H_{1}, H_{2}\right\} \subset \mathcal{D}$ with the smallest possible value of $l_{H_{1}}=l_{H_{2}}$ and the property

$$
I \subset H_{1} \odot H_{2} \supset J .
$$

The cubes $I$ and $J$ can be connected by the chain

$$
\widehat{I J}=\left\{H \in \mathcal{D}: I \subset H \subset I^{J} \text { or } J \subset H \subset J^{I}\right\} .
$$

We fix $\mu \in(0,1)$. For a function $f$ on a set $X \subset \mathbb{R}^{d}$ containing more than one point, we let

$$
|f|_{C^{\mu}(X)}=\sup _{x, y \in X: x \neq y} \frac{|f(x)-f(y)|}{|x-y|^{\mu}}, \quad\|f\|_{\text {Lip }}=\sup _{x, y \in X: x \neq y} \frac{|f(x)-f(y)|}{|x-y|} .
$$


We denote

$$
\begin{aligned}
\mathbb{R}_{+}^{n} & =\left\{x=\left(x^{\prime}, x_{n}\right) \in \mathbb{R}^{n}: x_{n}>0\right\}, \\
I^{Ð} & =\bar{I} \times\left[l_{I}, 2 l_{I}\right], \quad I \in \mathcal{D}, \\
\mathfrak{c}_{I}^{\bullet} & =\left(\mathfrak{c}_{I}, 3 l_{I} / 2\right) \quad \text { for the center } \mathfrak{c}_{I} \text { of the cube } I .
\end{aligned}
$$

Let $\mathcal{C}=C_{\text {loc }}^{\mu}\left(\mathbb{R}_{+}^{n}\right)$, that is, $\mathcal{C}$ consists of all real functions $f$ on $\mathbb{R}_{+}^{n}$ such that $|f|_{C^{\mu}\left(I^{\square}\right)}<\infty$ for each $I \in \mathcal{D}$. We let

$$
|f|_{I}=l_{I}^{\mu}|f|_{C^{\mu}\left(I^{\square}\right)}, \quad\|f\|_{I}=\|f\|_{L^{\infty}\left(I^{\square}\right)}+|f|_{I} .
$$

The estimate

$$
|f|_{I} \leqslant n l_{I}\|D f\|_{L^{\infty}\left(I^{\varpi}\right)}, \quad f \in C^{1}\left(I^{\square}\right)
$$

is obvious.

2.2. We introduce main notations related to the $Z$-parametrix $E(A ; x, y)$ of an arbitrary operator $A \in \mathcal{A}_{\lambda}^{\mu}$.

Let $\delta_{i j}$ be the Kronecker delta, $\Gamma(\cdot)$ be the Euler Gamma function, $\mathcal{A}$ be the set of all differential operators

$$
A=\sum_{i, j=1}^{n} a_{i j} D_{i j}
$$

with constant coefficients $a_{i j}=a_{j i} \in \mathbb{R}$. For $\lambda \geqslant 1$ we let

$$
\mathcal{A}_{\lambda}=\left\{A \in \mathcal{A}:\left(\forall \zeta \in \mathbb{R}^{n}\right) \lambda^{-1}|\zeta|^{2} \leqslant \sum_{i, j=1}^{n} a_{i j} \zeta_{i} \zeta_{j} \leqslant \lambda|\zeta|^{2}\right\} .
$$

We denote by $\mathfrak{z}_{I}$ the unique vertex of a cube $I \in \mathcal{D}$ possessing the property $\mathfrak{z}_{I} /\left(2 l_{I}\right) \in \mathbb{Z}^{n-1}$. Let

$$
\begin{array}{cc}
I^{\times}=\left\{\xi \in \overline{3 I}:\left|\xi-\mathfrak{z}_{I}\right|_{\infty} \leqslant 3 l_{I} / 2\right\} & \left(\Rightarrow I \subset \overline{2 I} \subset I^{\times} \subset \overline{3 I}\right), \\
I^{\bigotimes}=I^{\times} \times\left[3 l_{I} / 4,3 l_{I}\right] \quad & \left(\Rightarrow I^{\triangleright} \subset I^{\bigotimes}\right) .
\end{array}
$$

The symbol $\mathcal{A}^{\mu}$ stands for all operators (3) with real coefficients $a_{i j}=a_{j i} \in \mathcal{C}$. Hereafter $a_{i j}$ always stand for the coefficients of the operator $A \in \mathcal{A}$ or $A \in \mathcal{A}^{\mu}$. If $A \in \mathcal{A}^{\mu}$, then

$$
\begin{aligned}
& |A|_{I}=l_{I}^{\mu} \max _{i, j}\left|a_{i j}\right|_{C^{\mu}\left(I^{凶}\right)}, \\
& A[x]=\sum_{i, j=1}^{n} a_{i j}(x) D_{i j}, \quad x_{n}>0, \\
& \left.A f\right|_{x}=\left.A[x] f\right|_{x}, \quad f \in C^{2}\left(\mathbb{R}_{+}^{n}\right) .
\end{aligned}
$$

We let $\mathcal{A}_{\lambda}^{\mu}=\left\{A \in \mathcal{A}^{\mu}: A[x] \in \mathcal{A}_{\lambda}\right.$ for all $\left.x\right\}$.

For $I \in \mathcal{D}$ and $k \in \mathbb{N}_{0}$ by $I^{(k)}$ we denote the unique cube in $\mathcal{D}$ with the properties $I \subset I^{(k)}$ and $l_{I^{(k)}}=2^{k} l_{I}$. It is easy to construct the functions $\varphi_{k}: \mathbb{R}_{+}^{n} \rightarrow[0,1]$ in the class $C^{\infty}$ such that $\varphi_{0} \equiv 0$ and, as $k \geqslant 1$,

$$
\begin{aligned}
& \varphi_{k} \equiv 1 \text { on the set } \mathfrak{P}_{k} \equiv \overline{3 I^{(k-1)}} \times\left(0,3 l_{I^{(k-1)}}\right] \\
& \operatorname{supp} \varphi_{k} \subset \mathfrak{P}_{k}^{*} \equiv\left(5 I^{(k-1)}\right)^{\circ} \times\left(0,4 l_{\left.I^{(k-1)}\right)},\right. \\
& \left|D^{\alpha} \varphi_{k}\right| \leqslant c(\alpha) l_{I^{(k)}}^{-|\alpha|}, \quad \alpha \in \mathbb{N}_{0}^{n} .
\end{aligned}
$$

We also let $\mathfrak{Q}_{-1}=\mathfrak{P}_{0}=\mathfrak{P}_{0}^{*}=\varnothing$ and

$$
\mathfrak{Q}_{k}=\overline{3 I^{(k)}} \times\left(0,2 l_{I^{(k)}}\right], \quad k \geqslant 0 .
$$


It is obvious that

$$
\mathfrak{Q}_{k-1} \subset \mathfrak{P}_{k} \subset \mathfrak{P}_{k}^{*} \subset \mathfrak{Q}_{k}^{\circ}
$$

It is easy to check the existence of $C^{\infty}$-functions $\psi_{K}: \mathbb{R}_{+}^{n} \rightarrow[0,1]$ with the properties

$$
\begin{aligned}
& \operatorname{supp} \psi_{K} \subset \overline{\frac{3}{2} K} \times\left[\frac{3}{4} l_{K}, \frac{5}{2} l_{K}\right], \quad K \in \mathcal{D}, \\
& \sum_{K} \psi_{K}(x)=1, \quad x_{n}>0 \\
& \left|D^{\alpha} \psi_{K}\right| \leqslant c(\alpha) l_{K}^{-|\alpha|}, \quad \alpha \in \mathbb{N}_{0}^{n} .
\end{aligned}
$$

For $A \in \bigcup_{\lambda \geqslant 1} \mathcal{A}_{\lambda}$ and $x \neq 0$ we denote

$$
\begin{aligned}
& \operatorname{det}_{A}=\operatorname{det}\left(a_{i j}\right), \quad\left(b_{i j}\right)=\left(a_{i j}\right)^{-1}, \quad Q_{A}(x)=\sum_{i, j=1}^{n} b_{i j} x_{i} x_{j}, \\
& E_{A}(x)= \begin{cases}\frac{1}{4 \pi \sqrt{\operatorname{det}_{A}} \ln Q_{A}(x),} & n=2, \\
\frac{\Gamma(n / 2)}{(2-n) 2 \pi^{n / 2} \sqrt{\operatorname{det}_{A}} Q_{A}^{\frac{2-n}{2}}(x),} & n \geqslant 3 .\end{cases}
\end{aligned}
$$

For $x, y \in \mathbb{R}_{+}^{n}, x \neq y$, we let

$$
\begin{aligned}
& e_{n}^{A}=a_{n n}^{-1}\left\{a_{1 n} e_{1}+a_{2 n} e_{2}+\cdots+a_{n n} e_{n}\right\}, \\
& \widetilde{y}^{A}=y-y_{n} e_{n}^{A}, \quad \widetilde{y}_{A}=y-2 y_{n} e_{n}^{A} \\
& G_{A}(x, y)=E_{A}(x-y)-E_{A}\left(x-\widetilde{y}_{A}\right) .
\end{aligned}
$$

For $A \in \mathcal{A}_{\lambda}^{\mu}$ and $x, y \in \mathbb{R}_{+}^{n}, x \neq y$, we let

$$
E(A ; x, y)=\sum_{K} G_{A\left[c_{K}^{\bullet}\right]}(x, y) \psi_{K}(Z(x, y)),
$$

where

$$
Z(x, y)=x+\kappa|x-\widetilde{y}| e_{n}, \quad \kappa=\frac{1}{3 \sqrt{4 n+9}}, \quad \widetilde{y}=\left(y^{\prime},-y_{n}\right) .
$$

In [1] the parametrix $E(A ; x, y)$ was introduced with the constant $\kappa_{0}=\frac{1}{3 \sqrt{n+15}}$ instead of $\kappa$.

2.3. Let us write down the potential $\Phi_{f}$ and discrete Hölder estimates for it.

Theorem 1. Let $\lambda \geqslant 1,0<\mu<1$ and $A \in \mathcal{A}_{\lambda}^{\mu}$. Then for each function $f \in \mathrm{VL}(0)$, where

$$
\begin{aligned}
\operatorname{VL}(0) & =\left\{f \in \mathcal{C}:(\exists I \in \mathcal{D}) \sum_{J} \Gamma_{I J}^{(0, n)} l_{J}\|f\|_{J}<\infty\right\} \\
& =\left\{f \in \mathcal{C}:(\forall I \in \mathcal{D}) \sum_{J} \Gamma_{I J}^{(0, n)} l_{J}\|f\|_{J}<\infty\right\},
\end{aligned}
$$

the integral

$$
\Phi_{f}(x)=\int_{y_{n}>0} E(A ; x, y) f(y) d y
$$


converges absolutely and is twice continuously differentiable in $x$. We have $D^{\alpha} \Phi_{f} \in \mathcal{C}(|\alpha| \leqslant 2)$ and for each $I \in \mathcal{D}$

$$
\begin{aligned}
& l_{I}^{-1}\left\|\Phi_{f}\right\|_{I}+\left\|D \Phi_{f}\right\|_{I} \leqslant c(n, \lambda, \mu) \sum_{J} \Gamma_{I J}^{(0, n)} l_{J}\|f\|_{J} \\
& l_{I}^{-1}\left\|D_{n} \Phi_{f}-\mathbf{x}_{n}^{-1} \Phi_{f}\right\|_{I}+\left\|D^{2} \Phi_{f}\right\|_{I} \leqslant c(n, \lambda, \mu) \sum_{J} \Gamma_{I J}^{(0, n+1)}\|f\|_{J}, \\
& \left\|f-A \Phi_{f}\right\|_{I} \leqslant c(n, \lambda, \mu) \sum_{J} \Gamma_{I J}^{(0, n+1)}\|f\|_{J} \min \left\{1+|A|_{I}, \sum_{H: I \subset H \subset I^{J}}|A|_{H}\right\} .
\end{aligned}
$$

Remark. Here $\mathbf{x}_{n}^{-1}$ is the function $x \mapsto x_{n}^{-1}$.

Proof. All statements of the theorem, except the estimate for the norm $\left\|D_{n} \Phi_{f}-\mathbf{x}_{n}^{-1} \Phi_{f}\right\|_{I}$, were checked in [1, Thm. 5] for the parametrix $E(A ; x, y)$ defined by the constant $\kappa_{0}$ instead of $\kappa$. Due to the property $\kappa \leqslant \kappa_{0}$, the arguments can be extended to our parametrix with minor changes. This is why it remains to check the inequality:

$$
l_{I}^{-1}\left\|D_{n} \Phi_{f}-\mathbf{x}_{n}^{-1} \Phi_{f}\right\|_{I} \leqslant c(n, \lambda, \mu) \sum_{J} \Gamma_{I J}^{(0, n+1)}\|f\|_{J} .
$$

By (2), (4b) and 4c), for the function $\varphi_{1}$ in (4) we have

$$
\begin{aligned}
& \left\|\varphi_{1}\right\|_{J}+\left\|1-\varphi_{1}\right\|_{J} \leqslant 2+2 n l_{J}\left\|D \varphi_{1}\right\|_{L^{\infty}\left(J^{\varpi}\right)} \leqslant c_{1}(n), \\
& \left\|\varphi_{1} f\right\|_{J}+\left\|\left(1-\varphi_{1}\right) f\right\|_{J} \leqslant c_{1}\|f\|_{J}, \quad J \in \mathcal{D},
\end{aligned}
$$

and hence, $\varphi_{1} f \in \mathrm{VL}(0)$ and $\left(1-\varphi_{1}\right) f \in \mathrm{VL}(0)$. In the same way,

$$
\left\|\mathbf{x}_{n}^{-1} \Phi_{\varphi_{1} f}\right\|_{I} \leqslant\left\|\mathbf{x}_{n}^{-1}\right\|_{I}\left\|\Phi_{\varphi_{1} f}\right\|_{I} \leqslant c_{2}(n) l_{I}^{-1}\left\|\Phi_{\varphi_{1} f}\right\|_{I} .
$$

If $\left\|\varphi_{1} f\right\|_{J} \neq 0$, then $J^{\square} \cap \mathfrak{Q}_{1}^{\circ} \neq \varnothing$ in view of (4b) and (6), which implies $J^{\square} \subset \mathfrak{Q}_{1}$ and

$$
l_{I}^{-1} \Gamma_{I J}^{(0, n)} l_{J}=l_{I}^{-1} \Gamma_{I J}^{(0, n+1)}[I, J] \leqslant l_{I}^{-1} \Gamma_{I J}^{(0, n+1)}\left[I^{(1)}, J\right] \leqslant 4 \Gamma_{I J}^{(0, n+1)} .
$$

By (8) we conclude that

$$
\begin{aligned}
l_{I}^{-1}\left\|D_{n} \Phi_{\varphi_{1} f}-\mathbf{x}_{n}^{-1} \Phi_{\varphi_{1} f}\right\|_{I} & \leqslant l_{I}^{-1}\left\|D_{n} \Phi_{\varphi_{1} f}\right\|_{I}+c_{2} l_{I}^{-2}\left\|\Phi_{\varphi_{1} f}\right\|_{I} \\
& \leqslant c_{3}(n, \lambda, \mu) l_{I}^{-1} \sum_{J} \Gamma_{I J}^{(0, n)} l_{J}\left\|\varphi_{1} f\right\|_{J} \leqslant 4 c_{1} c_{3} \sum_{J} \Gamma_{I J}^{(0, n+1)}\|f\|_{J} .
\end{aligned}
$$

We assume that for each $x \in I^{\square}$ and $y \in J^{\square} \backslash \mathfrak{P}_{1}(J \in \mathcal{D})$, the functions

$$
\zeta_{K}(x, y)=G_{A\left[c_{K}^{\square}\right]}(x, y) \psi_{K}(Z(x, y)), \quad \zeta_{K}^{*}(x, y)=D_{x_{n}} \zeta_{K}(x, y)-x_{n}^{-1} \zeta_{K}(x, y)
$$

satisfy the inequalities

$$
\begin{aligned}
\left|\zeta_{K}(x, y)\right| & \leqslant c(n, \lambda) l_{I} \Gamma_{I J}^{(0, n)} l_{J}^{1-n} \\
\left|D_{x}^{\alpha} \zeta_{K}^{*}(x, y)\right| & \leqslant c(\alpha, \lambda) l_{I}^{1-|\alpha|} \Gamma_{I J}^{(0, n+1)} l_{J}^{-n}, \quad|\alpha| \leqslant 1 .
\end{aligned}
$$

Then by (4a), (7a) and the belonging $\left(1-\varphi_{1}\right) f \in \operatorname{VL}(0)$, the formula

$$
\Phi_{\left(1-\varphi_{1}\right) f}(x)=\int_{y_{n}>0}\left(\sum_{K} \zeta_{K}(x, y)\right)\left(1-\varphi_{1}(y)\right) f(y) d y
$$

leads us to the formula with an absolutely convergent series

$$
D^{\alpha}\left(D_{n} \Phi_{\left(1-\varphi_{1}\right) f}-\mathbf{x}_{n}^{-1} \Phi_{\left(1-\varphi_{1}\right) f}\right)(x)=\sum_{J, K} \int_{J^{\square}} D_{x}^{\alpha} \zeta_{K}^{*}(x, y)\left(1-\varphi_{1}(y)\right) f(y) d y,
$$


which together with (2), (7a) and the property $\int_{J^{\varpi}}|f| d y \leqslant l_{J}^{n}\|f\|_{J}$ yield

$$
l_{I}^{-1}\left\|D_{n} \Phi_{\left(1-\varphi_{1}\right) f}-\mathbf{x}_{n}^{-1} \Phi_{\left(1-\varphi_{1}\right) f}\right\|_{I} \leqslant c(n, \lambda) \sum_{J} \Gamma_{I J}^{(0, n+1)}\|f\|_{J} .
$$

In view of the result in the previous paragraph we obtain (11).

Let us check (13) and (14). For $x, y \in \mathbb{R}_{+}^{n}, x \neq y$, estimate (23) in [1] is of the form:

$$
\left|D_{x}^{\alpha} G_{B}(x, y)\right| \leqslant c(\alpha, \lambda) y_{n}|x-y|^{1-n-|\alpha|}, \quad(\alpha, B) \in \mathbb{N}_{0}^{n} \times \mathcal{A}_{\lambda} .
$$

Let $x \in I^{\square}$ and $y \in J^{\square} \backslash \mathfrak{P}_{1}(J \in \mathcal{D})$. Then

$$
[I, J] \leqslant 4\left|\left(x^{\prime}, \tau x_{n}\right)-y\right|_{\infty} \text { as } 0<\tau \leqslant 1,
$$

which is implied easily by the inequality $\left|\left(x^{\prime}, \tau x_{n}\right)-y\right|_{\infty}>l_{I}$. Hence,

$$
\left|G_{A\left[c_{K}^{\square}\right]}(x, y)\right| \leqslant\left|\int_{0}^{1} \frac{\partial}{\partial \tau} G_{A\left[c_{K}^{\square}\right]}\left(\left(x^{\prime}, \tau x_{n}\right), y\right) d \tau\right| \leqslant c(n, \lambda) l_{I} l_{J}[I, J]^{-n},
$$

which yields 13$)$. Let $\alpha \in\left\{0, e_{1}, \ldots, e_{n-1}\right\}$. By the Taylor formula,

$$
\begin{aligned}
& \zeta_{K}^{*}(x, y)=x_{n} \int_{0}^{1} \tau D^{2 e_{n}} \zeta_{K}\left(\left(x^{\prime}, \tau x_{n}\right), y\right) d \tau \\
& D_{x}^{\alpha} \zeta_{K}^{*}(x, y)=x_{n} \int_{0}^{1} \tau D^{\alpha+2 e_{n}} \zeta_{K}\left(\left(x^{\prime}, \tau x_{n}\right), y\right) d \tau \\
& D_{x_{n}} \zeta_{K}^{*}(x, y)=\int_{0}^{1}\left\{\tau D^{2 e_{n}}+x_{n} \tau^{2} D^{3 e_{n}}\right\} \zeta_{K}\left(\left(x^{\prime}, \tau x_{n}\right), y\right) d \tau
\end{aligned}
$$

where the derivatives $D^{\beta}$ are taken w.r.t. the first vector independent variable. By (15), (16), (7a), (7c) and the Leibnitz formula for $\bar{x}=\left(x^{\prime}, \tau x_{n}\right)$ we have

$$
\begin{aligned}
& \left|D_{\bar{x}}^{\beta} G_{A\left[c_{K}^{\bullet}\right]}(\bar{x}, y)\right| \leqslant c(\beta, \lambda) l_{J}[I, J]^{1-n-|\beta|}, \quad|\beta| \leqslant 3, \\
& \left|D_{\bar{x}}^{\beta} \psi_{K}(Z(\bar{x}, y))\right| \leqslant c(\beta)|\bar{x}-\widetilde{y}|^{-|\beta|} \leqslant c(\beta)[I, J]^{-|\beta|}, \\
& \left|D_{\bar{x}}^{\beta} \zeta_{K}(\bar{x}, y)\right| \leqslant c(\beta, \lambda) l_{J}[I, J]^{1-n-|\beta|} .
\end{aligned}
$$

Hence,

$$
\begin{aligned}
& \left|D_{x}^{\alpha} \zeta_{K}^{*}(x, y)\right| \leqslant c_{4}(\alpha, \lambda) l_{I} l_{J}[I, J]^{-n-1-|\alpha|} \leqslant c_{4} l_{I}^{1-|\alpha|} l_{J}[I, J]^{-n-1}, \\
& \left|D_{x_{n}} \zeta_{K}^{*}(x, y)\right| \leqslant c_{5}(n, \lambda)\left\{l_{J}[I, J]^{-n-1}+l_{I} l_{J}[I, J]^{-n-2}\right\} \leqslant 2 c_{5} l_{J}[I, J]^{-n-1},
\end{aligned}
$$

which coincides with (14). This completes the proof of inequality (11) and Theorem 1 .

2.4. Calculation of $\Phi_{A f}$. The proof of the following lemma is trivial.

Lemma 2. If $d \in \mathbb{N}, f \in C\left(\mathbb{R}^{d}\right)$ and $\sup _{\xi \in \mathbb{R}^{d}}|f(\xi)||\xi|^{d}<\infty$, then the limit

$$
\lim _{r \rightarrow \infty} \int_{|\xi-\mathfrak{x}|<r} f(\xi) d \xi
$$

either exists for all $\mathfrak{x} \in \mathbb{R}^{d}$ or does not exist for all $\mathfrak{x} \in \mathbb{R}^{d}$. In the former case, its value is independent of $\mathfrak{x}$.

We say that $D^{2} f \in \mathrm{VL}(0)$ if $f \in C_{\mathrm{loc}}^{2, \mu}\left(\mathbb{R}_{+}^{n}\right)$ and

$$
\sum_{J} \Gamma_{I J}^{(0, n)} l_{J}\left\|D^{2} f\right\|_{J}<\infty \text { for some } I \in \mathcal{D} \text {. }
$$

Let $\mathbb{P}_{1}^{n}$ be the space of all polynomials in $\mathbb{R}^{n}$ of degree at most one. By $\langle x, y\rangle$ we denote the scalar product $\sum_{i=1}^{n} x_{i} y_{i}$ in $\mathbb{R}^{n}$. 
Lemma 3. Let $D^{2} f \in \mathrm{VL}(0)$. Then

$$
\begin{aligned}
& t \nabla f(\cdot, t) \rightarrow 0 \text { in } L_{\mathrm{loc}}^{1}\left(\mathbb{R}^{n-1}\right) \text { as } t \downarrow 0, \\
& \text { in } L_{\mathrm{loc}}^{1}\left(\mathbb{R}^{n-1}\right) \text { there exists the limit } f(\cdot, 0+) .
\end{aligned}
$$

If $f(x)=\gamma(x)$ for large $|x|$ for some polynomial $\gamma \in \mathbb{P}_{1}^{n}$, then for each operator $A \in \bigcup_{\lambda \geqslant 1} \mathcal{A}_{\lambda}$ and points $x \in \mathbb{R}_{+}^{n}$ and $\mathfrak{x} \in \mathbb{R}^{n-1}$ we have

$$
\begin{aligned}
f(x) & -\int_{y_{n}>0} G_{A}(x, y) A f(y) d y \\
& =x_{n} \frac{\Gamma(n / 2)}{\pi^{n / 2} \sqrt{\operatorname{det}_{A}}} \lim _{r \rightarrow \infty} \int_{|\xi-\mathfrak{x}|<r} Q_{A}^{-n / 2}(x-(\xi, 0)) f(\xi, 0+) d \xi+\left\langle\nabla \gamma, e_{n}^{A}\right\rangle x_{n} .
\end{aligned}
$$

Remark. With no pedantry we write $f(\cdot, t)$ instead of $f((\cdot, t))$. The first integral in (20) exists by Theorem 1 since $G_{A}(x, y)=E(A ; x, y)$.

Proof. The condition $D^{2} f \in \mathrm{VL}(0)$ implies immediately that

$$
\int_{\Xi \times(0,1)} x_{n}\left|D^{2} f(x)\right| d x<\infty
$$

for each compact set $\Xi \subset \mathbb{R}^{n-1}$. For $0<t<1$ we have

$$
\begin{aligned}
t\|D f(\cdot, t)\|_{L^{1}(\Xi)} & \leqslant t\|D f(\cdot, 1)\|_{L^{1}(\Xi)}+t \int_{\Xi \times(t, 1)}\left|D^{2} f(x)\right| d x \\
& \leqslant t\|D f(\cdot, 1)\|_{L^{1}(\Xi)}+\int_{\Xi \times(t, \sqrt{t})} x_{n}\left|D^{2} f(x)\right| d x+\sqrt{t} \int_{\Xi \times(\sqrt{t}, 1)} x_{n}\left|D^{2} f(x)\right| d x,
\end{aligned}
$$

and as $t \rightarrow 0$, this proves (18). As $0<t_{1}<t_{2}<1$, the relations

$$
\begin{aligned}
& \left\|f\left(\cdot, t_{1}\right)-f\left(\cdot, t_{2}\right)\right\|_{L^{1}(\Xi)} \leqslant \int_{\Xi \times\left(t_{1}, t_{2}\right)}\left|D_{n} f(x)\right| d x \\
& \quad \leqslant t_{2}\left\|D_{n} f(\cdot, 1)\right\|_{L^{1}(\Xi)}+\int_{\Xi \times\left(t_{1}, t_{2}\right)} x_{n}\left|D_{n n} f(x)\right| d x+t_{2} \int_{\Xi \times\left(t_{2}, 1\right)}\left|D_{n n} f(x)\right| d x
\end{aligned}
$$

hold. These relations and the Cauchy convergence criterion give 119 ).

Let us prove that if the support supp $f$ is bounded under the assumptions of formula (20), then

$$
f(x)-\int_{y_{n}>0} G_{A}(x, y) A f(y) d y=x_{n} \frac{\Gamma(n / 2)}{\pi^{n / 2} \sqrt{\operatorname{det}_{A}}} \int_{\mathbb{R}^{n-1}} Q_{A}^{-n / 2}(x-(\xi, 0)) f(\xi, 0+) d \xi .
$$

In view of [1, (19)] and the formula

$$
\int_{\mathbb{R}^{n}} E_{A}(y-z) A \varphi(y) d y=\varphi(z), \quad \varphi \in C_{0}^{\infty}\left(\mathbb{R}^{n}\right)
$$

we get

$$
\begin{aligned}
& Q_{A}(x-y)-Q_{A}\left(x-\widetilde{y}_{A}\right)=-\frac{4 x_{n} y_{n}}{a_{n n}}=Q_{A}(y-x)-Q_{A}\left(y-\widetilde{x}_{A}\right), \\
& Q_{A}\left(x-\widetilde{y}_{A}\right)=Q_{A}\left(y-\widetilde{x}_{A}\right), \\
& G_{A}(x, y)=G_{A}(y, x), \\
& \int_{y_{n}>0} G_{A}(x, y) A \varphi(y) d y=\int_{y_{n}>0} G_{A}(y, x) A \varphi(y) d y=\varphi(x), \quad \varphi \in C_{0}^{\infty}\left(\mathbb{R}_{+}^{n}\right) .
\end{aligned}
$$


If the function $f$ is concentrated near the point $x$, by the regularization we have

$$
f(x)-\int_{y_{n}>0} G_{A}(x, y) A f(y) d y=0 .
$$

Hence, while checking (21), we can assume that $f \equiv 0$ in the vicinity of the point $x$. In this case, considering the integrals over the set $\left\{y: y_{n}>t\right\}$ and employing relations $\left.(15)\right|_{\alpha=0},(18$, (19) as well as the boundedness of $\operatorname{supp} f$, we obtain

$$
\begin{aligned}
-\int_{y_{n}>0} G_{A}(x, y) A f(y) d y & =\sum_{i, j=1}^{n} a_{i j} \int_{y_{n}>0} D_{y_{i}} G_{A}(x, y) D_{y_{j}} f(y) d y \\
& =\sum_{i, j=1}^{n} a_{i j} \int_{y_{n}>0} D_{y_{j}}\left\{D_{y_{i}} G_{A}(x, y) f(y)\right\} d y \\
& =-\sum_{i=1}^{n} a_{i n} \int_{\mathbb{R}^{n-1}} D_{y_{i}} G_{A}(x,(\xi, 0)) f(\xi, 0+) d \xi
\end{aligned}
$$

In view of $(22)$ we obtain

$$
\begin{aligned}
D_{y_{i}} G_{A}(x,(\xi, 0)) & =\left.C_{A} Q_{A}^{-n / 2}(x-y) D_{y_{i}}\left\{Q_{A}(x-y)-Q_{A}\left(x-\widetilde{y}_{A}\right)\right\}\right|_{y=(\xi, 0)} \\
& =-\frac{4 x_{n} \delta_{i n}}{a_{n n}} C_{A} Q_{A}^{-n / 2}(x-(\xi, 0)), \quad C_{A}=\frac{\Gamma(n / 2)}{4 \pi^{n / 2} \sqrt{\operatorname{det}_{A}}}
\end{aligned}
$$

This implies (21), that is, formula (20) with $\gamma=0$.

As $\gamma \neq 0$, we apply formula (21) to the function $f-\gamma$. Thanks to the identity

$$
\gamma(x)-\gamma\left(\widetilde{x}^{A}\right)=\left\langle\nabla \gamma, x_{n} e_{n}^{A}\right\rangle
$$

formula 20 will be proved if we establish the relation

$$
\gamma\left(\widetilde{x}^{A}\right)=x_{n} \frac{\Gamma(n / 2)}{\pi^{n / 2} \sqrt{\operatorname{det}_{A}}} \lim _{r \rightarrow \infty} \int_{|\xi-\mathfrak{x}|<r} Q_{A}^{-n / 2}(x-(\xi, 0)) \gamma(\xi, 0) d \xi .
$$

Expanding $\gamma(\xi, 0)$ into the powers of the variable $\eta \equiv \xi-\left(\widetilde{x}^{A}\right)^{\prime}$, we see that we need to check the identities

$$
\begin{aligned}
& x_{n} \frac{\Gamma(n / 2)}{\pi^{n / 2} \sqrt{\operatorname{det}_{A}}} \int_{\mathbb{R}^{n-1}} Q_{A}^{-n / 2}(x-(\xi, 0)) d \xi=1, \\
& \lim _{r \rightarrow \infty} \int_{\left|\eta+\left(\widetilde{x}^{A}\right)^{\prime}-\mathfrak{x}\right|<r} Q_{A}^{-n / 2}\left(x_{n} e_{n}^{A}-(\eta, 0)\right) \eta_{i} d \eta=0, \quad i=\overline{1, n-1} .
\end{aligned}
$$

The former identity is obtained by substituting the function $f=\varphi(\cdot / r)$ into (21), where $\varphi \in C_{0}^{\infty}\left(\mathbb{R}^{n}\right)$ and $\varphi \equiv 1$ in the vicinity of the origin and by passing then to limit as $r \rightarrow \infty$ taking into consideration the inequality

$$
\left|G_{A}(x, y)\right| \leqslant c(n, \lambda) x_{n}|x-y|^{1-n}
$$

implied by 15 and 24 . The second needed identity are yielded by the relations

$$
Q_{A}\left(x_{n} e_{n}^{A}-(\eta, 0)\right) \stackrel{\sqrt[23]{=}}{=} Q_{A}\left((\eta, 0)-x_{n} \widetilde{e_{n}^{A}}\right)=Q_{A}\left(x_{n} e_{n}^{A}+(\eta, 0)\right)
$$

and Lemma 2. This completes the proof of (25), 20) and the lemma.

The next result allows us to find approximately the derivatives $D^{\alpha} \Phi_{A f}$ of the potential $\Phi_{A f}$ and the expression $D_{n} \Phi_{A f}-\mathbf{x}_{n}^{-1} \Phi_{A f}$. 
Theorem 2. Let $\lambda \geqslant 1,0<\mu<1, A \in \mathcal{A}_{\lambda}^{\mu}, D^{2} f \in \operatorname{VL}(0), I \in \mathcal{D}$ and

$$
\Theta=\sum_{J} \Gamma_{I J}^{(0, n)} l_{J}\left(\sum_{H \in \overleftarrow{I J} \cup \overrightarrow{I J}}|A|_{H}\right) \mathcal{F}_{J}<\infty
$$

where

$$
\begin{aligned}
& \overleftarrow{I J}=\left\{H \in \mathcal{D}: I \subset H \subset I^{J} \& 12 l_{H}>\kappa l_{I^{J}}\right\} \\
& \overrightarrow{I J}=\left\{H \in \mathcal{D}: J \subset H \subset J^{I}\right\}, \\
& \mathcal{F}_{J}=\left\|D^{2} f\right\|_{J}+[I, J]^{-1} \sum_{H \in\left\{I^{J}\right\} \cup \overrightarrow{I J}} l_{H}\left\|D^{2} f\right\|_{H} .
\end{aligned}
$$

Then Af $\in \mathrm{VL}(0)$ and the integral

$$
\Phi_{A f}(x)=\int_{y_{n}>0} E(A ; x, y) A f(y) d y, \quad x \in \mathbb{R}_{+}^{n}
$$

converges absolutely. For $k \in \mathbb{N}_{0}$ and the functions $\left\{\varphi_{k}\right\}$ in (4) we denote

$$
\begin{aligned}
& \mathfrak{c}_{k}=\mathfrak{c}_{I^{(k)}}^{\square}, \quad A_{k}=A\left[\mathfrak{c}_{k}\right], \quad \gamma_{k}(x)=f\left(\mathfrak{c}_{k}\right)+\left\langle\nabla f\left(\mathfrak{c}_{k}\right), x-\mathfrak{c}_{k}\right\rangle, \\
& f_{k}=\varphi_{k} f+\left(1-\varphi_{k}\right) \gamma_{k}, \quad f_{(k)}=f_{k+1}-f_{k} .
\end{aligned}
$$

Then $D^{2} f_{k} \in \mathrm{VL}(0)$, the limits

$$
F_{k}(x)=\frac{\Gamma(n / 2)}{\pi^{n / 2} \sqrt{\operatorname{det}_{A_{k}}}} \lim _{r \rightarrow \infty} \int_{\left|\xi-\mathfrak{c}_{k}^{\prime}\right|<r} Q_{A_{k}}^{-n / 2}(x-(\xi, 0)) f_{(k)}(\xi, 0+) d \xi
$$

exist as $x \in I^{\square}$, the functions $F_{k}$ belong to $C^{2, \mu}\left(I^{\square}\right)$, the series

$$
F=\sum_{k=0}^{\infty} F_{k}
$$

converges absolutely in $C^{2, \mu}\left(I^{\square}\right)$, the scalar series

$$
\gamma^{\prime}=\sum_{k=0}^{\infty}\left\langle\nabla\left(\gamma_{k+1}-\gamma_{k}\right), e_{n}^{A_{k}}\right\rangle
$$

converges absolutely and the inequalities

$$
\begin{array}{r}
l_{I}^{-1}\left\|\mathcal{R}_{f}\right\|_{I}+\left\|D \mathcal{R}_{f}\right\|_{I} \leqslant c(n, \lambda, \mu) \Theta \\
l_{I}^{-1}\left\|D_{n} \mathcal{R}_{f}-\mathbf{x}_{n}^{-1} \mathcal{R}_{f}\right\|_{I}+\left\|D^{2} \mathcal{R}_{f}\right\|_{I} \leqslant c(n, \lambda, \mu) \Theta^{*}
\end{array}
$$

hold true, where

$$
\begin{aligned}
& \mathcal{R}_{f}=\Phi_{A f}-\Psi, \quad \Psi=f-\gamma_{0}-\mathbf{x}_{n} F-\gamma^{\prime} \mathbf{x}_{n}, \\
& \Theta^{*}=\sum_{J} \Gamma_{I J}^{(0, n+1)}\left(\sum_{H \in \overleftarrow{I J} \cup \overrightarrow{I J}}|A|_{H}\right) \mathcal{F}_{J} .
\end{aligned}
$$

Remark. The limits $f_{(k)}(\cdot, 0+)$ are treated in the sense of (19). The inequality

$$
\Theta^{*} \leqslant \Theta / l_{I}
$$

implies the finiteness of $\Theta^{*}$. 
Proof. It is obvious that $A f \in \mathcal{C}$. For each $J \in \mathcal{D}$ we have

$$
\begin{aligned}
& \left\|a_{i j}-a_{i j}\left(\mathfrak{c}_{J}^{\square}\right)\right\|_{J}=\left\|a_{i j}-a_{i j}\left(\mathfrak{c}_{J}^{\square}\right)\right\|_{L^{\infty}\left(J^{\square}\right)}+l_{J}^{\mu}\left|a_{i j}\right|_{C^{\mu}\left(J^{\square}\right)} \leqslant c(n)|A|_{J}, \\
& \|A f\|_{J} \leqslant\left\|A f-A\left[\mathfrak{c}_{J}^{\square}\right] f\right\|_{J}+\left\|A\left[\mathfrak{c}_{J}^{\square}\right] f\right\|_{J} \leqslant c(n)|A|_{J}\left\|D^{2} f\right\|_{J}+c(n, \lambda)\left\|D^{2} f\right\|_{J} .
\end{aligned}
$$

In view of the conditions $D^{2} f \in \operatorname{VL}(0)$ and $\Theta<\infty$ we obtain the belonging $A f \in \operatorname{VL}(0)$ and hence, the absolute convergence of the integral $\Phi_{A f}(x)$ by Theorem 1 .

Let $k \geqslant 0$ and $J^{\square} \subset \mathfrak{Q}_{k}$ (see (5)). Considering Taylor polynomials for the functions $f$ at the touching points for the cubes in the set $\left\{H^{\triangleright}: H \in \widehat{I^{(k)} J}\right\}$, by the inequalities

$$
\|\gamma\|_{L^{\infty}\left(J^{\square}\right)} \leqslant\|\gamma\|_{L^{\infty}\left(3\left(H^{\square}\right)\right)} \leqslant c(n)\|\gamma\|_{L^{\infty}\left(H^{\square}\right)}, \quad \gamma \in \mathbb{P}_{1}^{n},
$$

and the Taylor formula we obtain the estimates

$$
\begin{aligned}
& \left\|f-\gamma_{k}\right\|_{L^{\infty}\left(J^{\square}\right)} \leqslant c(n) \sum_{H \in \widehat{I^{(k)} J}} l_{H}^{2}\left\|D^{2} f\right\|_{L^{\infty}\left(H^{\square}\right)}, \\
& \left\|D\left(f-\gamma_{k}\right)\right\|_{L^{\infty}\left(J^{\square}\right)} \leqslant c(n) \sum_{H \in \widehat{I^{(k)} J}} l_{H}\left\|D^{2} f\right\|_{L^{\infty}\left(H^{\square}\right)} .
\end{aligned}
$$

In view of relations (2), $\left|D^{2} \gamma_{k}\right| \equiv 0$ and $\left(I^{(k)}\right)^{J}=I^{(k)}$ we conclude that

$$
\begin{aligned}
& \left\|f-\gamma_{k}\right\|_{J} \leqslant c(n) \sum_{H \in \widehat{I^{(k)} J}} l_{H}^{2}\left\|D^{2} f\right\|_{H}, \\
& \left\|D\left(f-\gamma_{k}\right)\right\|_{J} \leqslant c(n) \sum_{H \in \widehat{I^{(k)} J}} l_{H}\left\|D^{2} f\right\|_{H}, \\
& l_{H} \leqslant l_{I^{(k)}} \quad \& \quad[I, J] \leqslant\left[I^{(k)}, J\right] \leqslant 2 l_{I^{(k)}}, \\
& l_{I^{(k)}}^{-2}\left\|f-\gamma_{k}\right\|_{J}+l_{I^{(k)}}^{-1}\left\|D\left(f-\gamma_{k}\right)\right\|_{J} \leqslant c(n)[I, J]^{-1} \sum_{H \in \widehat{I^{(k)} J}} l_{H}\left\|D^{2} f\right\|_{H} .
\end{aligned}
$$

Let us estimate $\left\|D^{2}\left(f-f_{k}\right)\right\|_{J}$ and $\left\|D^{2} f_{(k)}\right\|_{J}$. We write

$$
f-f_{k}=\left(1-\varphi_{k}\right)\left(f-\gamma_{k}\right), \quad f_{(k)}=f_{k+1}-f_{k}=\left\{f-f_{k}\right\}-\left\{f-f_{k+1}\right\} .
$$

If $J^{\square} \subset \mathfrak{Q}_{k} \backslash \mathfrak{Q}_{k-1}^{\circ}$, then $l_{J} \leqslant l_{I^{(k)}}$ and $\widehat{I^{(k)} J}=\left\{I^{J}\right\} \cup \overrightarrow{I J}$. By (2), 4c), 28) and the Leibnitz formula, this implies

$$
l_{I^{(k)}}^{2}\left\|D^{2}\left(1-\varphi_{k}\right)\right\|_{J}+l_{I^{(k)}}\left\|D\left(1-\varphi_{k}\right)\right\|_{J}+\left\|1-\varphi_{k}\right\|_{J} \leqslant c(n), \quad\left\|D^{2}\left(f-f_{k}\right)\right\|_{J} \leqslant c_{1}(n) \mathcal{F}_{J} .
$$

By 4a), 4b) and (6),

$$
\begin{aligned}
f-f_{k} \equiv 0 & \text { on the set } \mathfrak{Q}_{k-1} \\
\left|D^{2} f_{k}\right| \equiv 0 & \text { on the set } \mathbb{R}_{+}^{n} \backslash \mathfrak{Q}_{k}^{\circ} \\
\left|D^{2} f_{(k)}\right| \equiv 0 & \text { on the set } \mathbb{R}_{+}^{n} \backslash \mathfrak{Q}_{k+1}^{\circ}
\end{aligned}
$$

Therefore,

$$
\begin{aligned}
\left\|D^{2}\left(f-f_{k}\right)\right\|_{J} \leqslant \begin{cases}0, & J^{\varpi} \subset \mathfrak{Q}_{k-1}, \\
c_{1} \mathcal{F}_{J}, & J^{\square} \subset \mathfrak{Q}_{k} \backslash \mathfrak{Q}_{k-1}^{\circ}, \\
\left\|D^{2} f\right\|_{J} \leqslant \mathcal{F}_{J}, & J^{\square} \subset \mathbb{R}_{+}^{n} \backslash \mathfrak{Q}_{k}^{\circ},\end{cases} \\
\left\|D^{2} f_{(k)}\right\|_{J} \leqslant \begin{cases}\left(c_{1}+1\right) \mathcal{F}_{J}, & J^{\square} \subset \mathfrak{Q}_{k+1} \backslash \mathfrak{Q}_{k-1}^{\circ}, \\
0 & \text { otherwise. }\end{cases}
\end{aligned}
$$


In view of $\left[9\right.$, Eq. (25e)], we have $\left[I^{J}, J\right] \leqslant 3[I, J]$ and hence, for each $\alpha, \beta \in \mathbb{R}$

$$
\Gamma_{I J}^{(\alpha, \beta)} \leqslant \max \left\{1,3^{\alpha+\beta}\right\} \Gamma_{I H}^{(\alpha, \beta)} \Gamma_{H J}^{(\alpha, \beta)}, \quad H \in \widehat{I J} .
$$

Let $\alpha \in \mathbb{R}, \beta>n-1$ and $H \in \overleftarrow{I J} \cup \overrightarrow{I J}$. Then

$$
\begin{aligned}
& {[H, J] \leqslant\left[I^{J}, J^{I}\right] \leqslant 2 l_{I^{J}}<24 \kappa^{-1} l_{H} \quad \text { as } H \in \overleftarrow{I J}, \quad[H, J]=l_{H} \quad \text { as } H \in \overrightarrow{I J},} \\
& \Gamma_{H J}^{(\alpha, \beta)} \leqslant c(n, \alpha) \Gamma_{H J}^{\left(\alpha_{1}, \beta\right)}, \quad \alpha_{1}=\max \{\alpha, 1\} .
\end{aligned}
$$

By (31) and Lemma 1, for each $H \in \mathcal{D}$ we obtain

$$
\sum_{J: H \in \overleftarrow{I J} \cup \overrightarrow{I J}} \Gamma_{I J}^{(\alpha, \beta)} \leqslant c(n, \alpha, \beta) \Gamma_{I H}^{(\alpha, \beta)} \sum_{J} \Gamma_{H J}^{\left(\alpha_{1}, \beta\right)} \leqslant c(n, \alpha, \beta) \Gamma_{I H}^{(\alpha, \beta)} .
$$

Hence,

$$
\begin{gathered}
\sum_{J} \Gamma_{I J}^{(0, n)} l_{J}[I, J]^{-1} \sum_{H \in\left\{I^{J}\right\} \cup \overrightarrow{I J}} l_{H}\left\|D^{2} f\right\|_{H} \\
\leqslant \sum_{H} l_{H}\left\|D^{2} f\right\|_{H} \sum_{J: H \in \overleftarrow{I J} \cup \overrightarrow{I J}} \Gamma_{I J}^{(0, n+1)} \\
\leqslant c(n) \sum_{H} \Gamma_{I H}^{(0, n+1)} l_{H}\left\|D^{2} f\right\|_{H}, \\
\theta:=\sum_{J} \Gamma_{I J}^{(0, n)} l_{J} \mathcal{F}_{J} \leqslant c(n) \sum_{J} \Gamma_{I J}^{(0, n)} l_{J}\left\|D^{2} f\right\|_{J}<\infty .
\end{gathered}
$$

In view of 29$)$ we conclude that $D^{2}\left(f-f_{k}\right), D^{2} f_{k}, D^{2} f_{(k)} \in \operatorname{VL}(0)$.

In what follows we suppose that $x \in I^{\square}$. Due to (30) we have

$$
\begin{aligned}
& \left\|A f_{(k)}\right\|_{J} \leqslant c(n, \lambda)\left(|A|_{J}+1\right)\left\|D^{2} f_{(k)}\right\|_{J}, \quad\left\|A_{k} f_{(k)}\right\|_{J} \leqslant c(n, \lambda)\left\|D^{2} f_{(k)}\right\|_{J}, \\
& \sum_{J} \Gamma_{I J}^{(0, n)} l_{J}\left\{\left\|A f_{(k)}\right\|_{J}+\left\|A_{k} f_{(k)}\right\|_{J}\right\} \leqslant c_{2}(n, \lambda) \sum_{J} \Gamma_{I J}^{(0, n)} l_{J}\left(|A|_{J}+1\right) \mathcal{F}_{J} \leqslant c_{2}\{\Theta+\theta\}<\infty .
\end{aligned}
$$

Hence, $A f_{(k)} \in \mathrm{VL}(0)$ and $A_{k} f_{(k)} \in \mathrm{VL}(0)$, so, the potentials

$$
\begin{aligned}
& \Phi_{k}(x)=\int_{y_{n}>0} E(A ; x, y) A f_{(k)}(y) d y, \\
& \Phi_{k}^{\prime}(x)=\int_{y_{n}>0} E(A ; x, y) A_{k} f_{(k)}(y) d y, \\
& \Phi_{k}^{\prime \prime}(x)=\int_{y_{n}>0} G_{A_{k}}(x, y) A_{k} f_{(k)}(y) d y
\end{aligned}
$$

are well-defined. In view of (8), (9), (29) and (30), the series

$$
\Phi=\sum_{k=0}^{\infty} \Phi_{k}, \quad \Phi^{\prime}=\sum_{k=0}^{\infty} \Phi_{k}^{\prime}, \quad \Phi^{\prime \prime}=\sum_{k=0}^{\infty} \Phi_{k}^{\prime \prime}
$$

converge absolutely in $C^{2, \mu}\left(I^{\square}\right)$, and the potential $\Phi_{A\left(f-f_{k}\right)}$ tends to zero as $k \rightarrow \infty$ in $C^{2, \mu}\left(I^{\square}\right)$. Bearing in mind the relation $f_{0}=\gamma_{0} \in \mathbb{P}_{1}^{n}$, on $I^{\square}$ we get

$$
\Phi_{A f}=\Phi_{A\left(f-f_{q}\right)}+\sum_{k=0}^{q-1} \Phi_{k}=\lim _{q \rightarrow \infty}\left(\Phi_{A\left(f-f_{q}\right)}+\sum_{k=0}^{q-1} \Phi_{k}\right)=\Phi .
$$

By Lemma 3, the limits $F_{k}(x)$ exist and

$$
f_{(k)}-\Phi_{k}^{\prime \prime}=\mathbf{x}_{n} F_{k}+\left\langle\nabla\left(\gamma_{k+1}-\gamma_{k}\right), e_{n}^{A_{k}}\right\rangle \mathbf{x}_{n}
$$


In particular, $F_{k} \in C^{2, \mu}\left(I^{\varpi}\right)$. By the Taylor formula

$$
\begin{gathered}
\left|\nabla\left(\gamma_{k+1}-\gamma_{k}\right)\right| \leqslant c(n) l_{I^{(k)}}\left\{\left\|D^{2} f\right\|_{I^{(k)}}+\left\|D^{2} f\right\|_{I^{(k+1)}}\right\}, \\
\sum_{k=0}^{\infty}\left|\left\langle\nabla\left(\gamma_{k+1}-\gamma_{k}\right), e_{n}^{A_{k}}\right\rangle\right| \leqslant c(n, \lambda) \sum_{J} \Gamma_{I J}^{(0, n)} l_{J}\left\|D^{2} f\right\|_{J}<\infty
\end{gathered}
$$

that is, the series $\gamma^{\prime}$ converges absolutely. The absolute convergence of the series $F$ follows the relation $\left.f_{(k)}\right|_{I^{\square}} \equiv 0(k \geqslant 1)$ and the absolute convergence of the series $\Phi^{\prime \prime}$ and $\gamma^{\prime}$. On the cube $I^{\square}$, the identities

$$
\Psi=f-\gamma_{0}-\sum_{k=0}^{\infty}\left\{f_{(k)}-\Phi_{k}^{\prime \prime}\right\}=f-\gamma_{0}-f_{(0)}+\Phi^{\prime \prime}=\Phi^{\prime \prime}, \quad \mathcal{R}_{f}=\Phi-\Phi^{\prime \prime}
$$

hold true.

It remains to check inequalities (26). If $J^{\square} \subset \mathfrak{Q}_{k+1} \backslash \mathfrak{Q}_{k-1}^{\circ}$, where $k \geqslant 0$, then $I^{J}=I^{(k)}$ or $I^{J}=I^{(k+1)}$, so that $\mathfrak{c}_{k} \in\left(I^{J}\right)^{\bigotimes}$. By analogy with $(27)$ we have

$$
\begin{aligned}
& \left\|a_{i j}-a_{i j}\left(\mathfrak{c}_{k}\right)\right\|_{J} \leqslant c(n) \sum_{H \in\left\{I^{J}\right\} \cup \overrightarrow{I J}}|A|_{H}, \\
& \left\|\left(A-A_{k}\right) f_{(k)}\right\|_{J} \leqslant c(n)\left(\sum_{H \in\left\{I^{J}\right\} \cup \overrightarrow{I J}}|A|_{H}\right)\left\|D^{2} f_{(k)}\right\|_{J} .
\end{aligned}
$$

In view of $(30)$ and by Theorem 1 we obtain

$$
\begin{aligned}
& l_{I}^{-1}\left\|\Phi_{k}-\Phi_{k}^{\prime}\right\|_{I}+\left\|D\left(\Phi_{k}-\Phi_{k}^{\prime}\right)\right\|_{I} \leqslant c(n, \lambda, \mu) \Theta_{k} \\
& l_{I}^{-1}\left\|D_{n}\left(\Phi_{k}-\Phi_{k}^{\prime}\right)-\mathbf{x}_{n}^{-1}\left(\Phi_{k}-\Phi_{k}^{\prime}\right)\right\|_{I}+\left\|D^{2}\left(\Phi_{k}-\Phi_{k}^{\prime}\right)\right\|_{I} \leqslant c(n, \lambda, \mu) \Theta_{k}^{*}
\end{aligned}
$$

where

$$
\begin{aligned}
& \Theta_{k}=\sum_{J: J \boxminus \subset \mathfrak{Q}_{k+1} \backslash \mathfrak{Q}_{k-1}^{\circ}} \Gamma_{I J}^{(0, n)} l_{J}\left(\sum_{H \in \overleftarrow{I J} \cup \overrightarrow{I J}}|A|_{H}\right) \mathcal{F}_{J}, \\
& \Theta_{k}^{*}=\sum_{J: J^{\square} \subset \mathfrak{Q}_{k+1} \backslash \mathfrak{Q}_{k-1}^{\circ}} \Gamma_{I J}^{(0, n+1)}\left(\sum_{H \in \overleftarrow{I J} \cup \overrightarrow{I J}}|A|_{H}\right) \mathcal{F}_{J} .
\end{aligned}
$$

By this and the convergence of the series $\Phi$ and $\Phi^{\prime}$ we conclude that

$$
\begin{aligned}
& l_{I}^{-1}\left\|\Phi-\Phi^{\prime}\right\|_{I}+\left\|D\left(\Phi-\Phi^{\prime}\right)\right\|_{I} \leqslant c(n, \lambda, \mu) \Theta, \\
& l_{I}^{-1}\left\|D_{n}\left(\Phi-\Phi^{\prime}\right)-\mathbf{x}_{n}^{-1}\left(\Phi-\Phi^{\prime}\right)\right\|_{I}+\left\|D^{2}\left(\Phi-\Phi^{\prime}\right)\right\|_{I} \leqslant c(n, \lambda, \mu) \Theta^{*} .
\end{aligned}
$$

We let

$$
\mathcal{H}_{k}=\left\{K \in \mathcal{D}: \mathfrak{c}_{K}^{\square} \in\left(I^{(j)}\right)^{\bigotimes} \text { for some } j \in \mathbb{N}_{0}, k-\log _{2}(6 / \kappa)<j \leqslant k\right\} .
$$

For each $(k, K) \in \mathbb{N}_{0} \times \mathcal{D}$ we shall show that

$$
\text { if } \psi_{K}(Z(x, y)) A_{k} f_{(k)}(y) \neq 0 \text { for some } y \in \mathbb{R}_{+}^{n} \text {, then } K \in \mathcal{H}_{k} .
$$


Suppose the assumption in (34). Then $y \in \mathfrak{Q}_{k+1} \backslash \mathfrak{Q}_{k-1}$ by (30) and hence,

$$
\begin{aligned}
&|x-\widetilde{y}|>x_{n} \geqslant l_{I}, \quad k=0, \\
&|x-\widetilde{y}| \geqslant \max \left\{\left|x^{\prime}-y^{\prime}\right|_{\infty}, y_{n}\right\}>l_{I^{(k-1)}}, \quad k \neq 0, \\
&|x-\widetilde{y}|^{2} \leqslant(n-1)\left|x^{\prime}-y^{\prime}\right|_{\infty}^{2}+\left(x_{n}+y_{n}\right)^{2} \\
& \leqslant 16(n-1) l_{I^{(k)}}^{2}+\left(2 l_{I}+4 l_{I^{(k)}}\right)^{2}<\kappa^{-2} l_{I^{(k)}}^{2}, \\
& l_{I}<l_{I}+\frac{\kappa}{2} l_{I^{(k)}}<Z(x, y)_{n}=x_{n}+\kappa|x-\widetilde{y}|<2 l_{I}+l_{I^{(k)}} \leqslant 3 l_{I^{(k)}} .
\end{aligned}
$$

This is why there exists $j$ such that $0 \leqslant j \leqslant k$ and

$$
Z(x, y) \in\left[\overline{I^{(j)}} \times\left(l_{I^{(j)}}, 3 l_{I^{(j)}}\right)\right] \cap \operatorname{supp} \psi_{K} .
$$

We have

$$
\frac{\kappa}{2} l_{I^{(k)}}<Z(x, y)_{n}<3 l_{I^{(j)}},
$$

and hence, $k-\log _{2}(6 / \kappa)<j$. By $(7 \mathrm{a})$

$$
\overline{I^{(j)}} \cap \overline{\frac{3}{2} K} \neq \varnothing \quad \& \quad \frac{1}{2} \leqslant \frac{l_{I^{(j)}}}{l_{K}} \leqslant 2 .
$$

It is easy to confirm that this implies the belonging $\mathfrak{c}_{K}^{\square} \in\left(I^{(j)}\right)^{\square}$. The proof of (34) is complete.

It follows from relations $(7 \mathrm{~b}),(30)$ and $(34)$ that

$$
\Phi_{k}^{\prime}(x)-\Phi_{k}^{\prime \prime}(x)=\sum_{K \in \mathcal{H}_{k}} \int_{\mathfrak{Q}_{k+1} \backslash \mathfrak{Q}_{k-1}^{\circ}}\left(G_{A\left[\mathfrak{c}_{K}^{\square}\right]}(x, y)-G_{A_{k}}(x, y)\right) \psi_{K}(Z(x, y)) A_{k} f_{(k)}(y) d y .
$$

If $J^{\square} \subset \mathfrak{Q}_{k+1} \backslash \mathfrak{Q}_{k-1}^{\circ}$, then $I^{J}=I^{(k)}$ or $I^{J}=I^{(k+1)}$ and therefore,

$$
I \subset I^{(j)} \subset I^{(k)} \subset I^{J} \quad \& \quad l_{I^{(j)}}>\frac{\kappa}{6} l_{I^{(k)}} \geqslant \frac{\kappa}{12} l_{I^{J}} \quad \& \quad I^{(j)} \in \overleftarrow{I J}
$$

for each index $j$ in the definition of the set $\mathcal{H}_{k}$. This is why

$$
\begin{gathered}
\max _{i, j=\overline{1, n}} \max _{K \in \mathcal{H}_{k}}\left|a_{i j}\left(\mathfrak{c}_{K}^{\square}\right)-a_{i j}\left(\mathfrak{c}_{k}\right)\right| \leqslant c(n) \sum_{H \in \overleftarrow{I J}}|A|_{H}, \quad J^{\square} \subset \mathfrak{Q}_{k+1} \backslash \mathfrak{Q}_{k-1}^{\circ}, \\
\left\|A_{k} f_{(k)}\right\|_{J} \leqslant c(n, \lambda) \mathcal{F}_{J},
\end{gathered}
$$

where the second inequality is implied trivially by (30). By a simple modification of the constructions in work [1], from (35) and (36) we obtain the estimates

$$
\begin{aligned}
& l_{I}^{-1}\left\|\Phi_{k}^{\prime}-\Phi_{k}^{\prime \prime}\right\|_{I}+\left\|D\left(\Phi_{k}^{\prime}-\Phi_{k}^{\prime \prime}\right)\right\|_{I} \leqslant c(n, \lambda, \mu) \Theta_{k}, \\
& \left\|D^{2}\left(\Phi_{k}^{\prime}-\Phi_{k}^{\prime \prime}\right)\right\|_{I} \leqslant c(n, \lambda, \mu) \Theta_{k}^{*} .
\end{aligned}
$$

In [1, Subsect. 2.1], an estimate for the derivatives of the Green functions was proved (see (15)), which was applied for estimating the norms of $\left\|D^{\alpha} \Phi\right\|_{I}$ in [1, Subsect. 2.2], where the function $\Phi$ is similar to the potential $\Phi_{f}$. At the same time, in [1, Subsect. 2.1], there was obtained an estimate for the derivatives of the difference $G_{B_{1}}-G_{B_{2}}$ applied then in [1, Subsect. 2.2] for estimating the norm $\|f-A \Phi\|_{I}$ of the error $f-A \Phi$. These two lines can be easily combined to obtain inequalities (37). By (12) and (37a)

$$
l_{I}^{-1}\left\|D_{n}\left(\Phi_{0}^{\prime}-\Phi_{0}^{\prime \prime}\right)-\mathbf{x}_{n}^{-1}\left(\Phi_{0}^{\prime}-\Phi_{0}^{\prime \prime}\right)\right\|_{I} \leqslant c(n, \lambda, \mu) \Theta_{0}^{*} .
$$

This is why, if we establish the inequality

$$
l_{I}^{-1}\left\|D_{n}\left(\Phi_{k}^{\prime}-\Phi_{k}^{\prime \prime}\right)-\mathbf{x}_{n}^{-1}\left(\Phi_{k}^{\prime}-\Phi_{k}^{\prime \prime}\right)\right\|_{I} \leqslant c(n, \lambda) \Theta_{k}^{*} \quad(k \geqslant 1),
$$

then the convergence of the series $\Phi^{\prime}$ and $\Phi^{\prime \prime}$ in $C^{2, \mu}\left(I^{\square}\right)$ and the relation $\mathcal{R}_{f}=\Phi-\Phi^{\prime \prime}$ (on $I^{\square}$ ) and (33) will imply required estimates (26). 
Let $k \geqslant 1, J^{\square} \subset \mathfrak{Q}_{k+1} \backslash \mathfrak{Q}_{k-1}^{\circ}, \bar{x}=\left(x^{\prime}, \tau x_{n}\right)$ for $0<\tau \leqslant 1, y \in J^{\square} \backslash \mathfrak{P}_{1}$ and $K \in \mathcal{H}_{k}$. By equation (23') in [1], (16) and (36a) we get

$$
\begin{aligned}
& \left|D_{\bar{x}}^{\beta}\left(G_{A\left[\mathfrak{c}_{K}^{\square}\right]}(\bar{x}, y)-G_{A_{k}}(\bar{x}, y)\right)\right| \leqslant c(\beta, \lambda) y_{n}|\bar{x}-y|^{1-n-|\beta|} \sum_{H \in \overleftarrow{I J}}|A|_{H} \\
& \leqslant c(\beta, \lambda) l_{J}[I, J]^{1-n-|\beta|} \sum_{H \in \overleftarrow{I J}}|A|_{H}, \quad|\beta| \leqslant 3,
\end{aligned}
$$

which can be considered as an analogue of inequality (17d). Reproducing (17) for the functions

$$
\begin{aligned}
& \delta_{K}(\bar{x}, y)=\left(G_{A\left[\mathfrak{c}_{K}^{\square}\right]}(\bar{x}, y)-G_{A_{k}}(\bar{x}, y)\right) \psi_{K}(Z(\bar{x}, y)), \\
& \delta_{K}^{*}(x, y)=D_{x_{n}} \delta_{K}(x, y)-x_{n}^{-1} \delta_{K}(x, y),
\end{aligned}
$$

we arrive at the estimates

$$
\begin{aligned}
& \left|D_{\bar{x}}^{\beta} \delta_{K}(\bar{x}, y)\right| \leqslant c(\beta, \lambda) l_{J}[I, J]^{1-n-|\beta|} \sum_{H \in \overleftarrow{I J}}|A|_{H}, \\
& \left|D_{x}^{\alpha} \delta_{K}^{*}(x, y)\right| \leqslant c(\alpha, \lambda) l_{I}^{1-|\alpha|} l_{J}[I, J]^{-n-1} \sum_{H \in \overleftarrow{I J}}|A|_{H}, \quad \alpha \in\left\{0, e_{1}, \ldots, e_{n-1}\right\} \\
& \left|D_{x_{n}} \delta_{K}^{*}(x, y)\right| \leqslant c(n, \lambda) l_{J}[I, J]^{-n-1} \sum_{H \in \overleftarrow{I J}}|A|_{H} .
\end{aligned}
$$

In view of (4a), (35), (39) and the belonging $A_{k} f_{(k)} \in \mathrm{VL}(0)$, the formula

$$
\begin{aligned}
D^{\alpha}\left(D_{n}\left(\Phi_{k}^{\prime}-\Phi_{k}^{\prime \prime}\right)\right. & \left.-\mathbf{x}_{n}^{-1}\left(\Phi_{k}^{\prime}-\Phi_{k}^{\prime \prime}\right)\right)(x) \\
& =\sum_{J, K: J^{\square} \subset \mathfrak{Q}_{k+1} \backslash \mathfrak{Q}_{k-1}^{\circ}} \text { and } K \in \mathcal{H}_{k} \\
\int_{J \square \backslash \mathfrak{P}_{1}} & D_{x}^{\alpha} \delta_{K}^{*}(x, y) A_{k} f_{(k)}(y) d y, \quad|\alpha| \leqslant 1
\end{aligned}
$$

holds true, where the series converges absolutely. Hence, in view of (2), (36b), 40) and (41) we obtain estimate $(38)$. This completes the proof of $(26)$ and of the theorem.

\section{Standard Set and CALCUlations With the Potential $\Phi_{L}$}

3.1. Standard set and potential $\Phi_{A w}$. To a Lipschitz function $\omega: \mathbb{R}^{n-1} \rightarrow \mathbb{R}$ we associate its overgraph $\Omega$ and the approximation numbers $b_{I}$ :

$$
\begin{aligned}
& \Omega=\left\{x=\left(x^{\prime}, x_{n}\right) \in \mathbb{R}^{n}: x_{n}>\omega\left(x^{\prime}\right)\right\}, \\
& b_{I}=l_{I}^{-\frac{n+1}{2}}\left(\min _{\gamma \in \mathbb{P}_{1}^{n-1}} \int_{5 I}|\omega-\gamma|^{2} d \xi\right)^{1 / 2}, \quad I \in \mathcal{D} .
\end{aligned}
$$

We introduce a series of auxiliary notions needed for studying harmonic functions in the domain $\Omega$ by straightening this domain.

Theorem 3. Given $K \in \mathcal{D}$, let $\gamma_{K} \in \mathbb{P}_{1}^{n-1}$ be a polynomial with the property

$$
\int_{K}\left|\omega-\gamma_{K}\right|^{2} d \xi=\min _{\gamma \in \mathbb{P}_{1}^{n-1}} \int_{K}|\omega-\gamma|^{2} d \xi
$$

For the partition of unity $\left\{\psi_{K}\right\}$ in (7) we let

$$
w(x)=\sum_{K} \psi_{K}(x) \gamma_{K}\left(x^{\prime}\right), \quad x \in \mathbb{R}_{+}^{n} .
$$

Then the function $w$ belongs to $C^{\infty}\left(\mathbb{R}_{+}^{n}\right)$, is Lipschitz and

$$
w(\xi, 0+)=\omega(\xi), \quad \xi \in \mathbb{R}^{n-1} .
$$


We choose a constant $\theta \geqslant\|\omega\|_{\text {Lip. }}$. Then for each $I \in \mathcal{D}$

$$
\begin{aligned}
& \left|\nabla \gamma_{I}\right| \leqslant c(n) \theta, \\
& b_{I} \leqslant c(n) \theta, \\
& \left\|D^{\alpha} w\right\|_{L^{\infty}\left(I^{\otimes}\right)} \leqslant c(\alpha) l_{I}^{1-|\alpha|} b_{I}, \quad \alpha \notin\left\{0, e_{1}, \ldots, e_{n-1}\right\}, \\
& \left\|D^{\alpha} w\right\|_{L^{\infty}\left(I^{\otimes}\right)} \leqslant c(\alpha) l_{I}^{1-|\alpha|} \theta, \quad \alpha \neq 0 .
\end{aligned}
$$

There exists $W=c(n, \theta)$ such that for the mapping $\mathbf{x}^{\prime}: x \mapsto x^{\prime}$

$$
\begin{aligned}
& \left\|\omega-\gamma_{I}\right\|_{L^{\infty}(5 I)} \leqslant W l_{I} / 3, \quad I \in \mathcal{D}, \\
& \left\|w-\gamma_{I} \circ \mathbf{x}^{\prime}\right\|_{L^{\infty}\left(I^{\boxplus)}\right.} \leqslant W l_{I} / 3, \quad I \in \mathcal{D},
\end{aligned}
$$

the mapping $g: \mathbb{R}_{+}^{n} \rightarrow \mathbb{R}^{n}$ of the form $g(x)=\left(x^{\prime}, g_{n}(x)\right)$ with the function

$$
g_{n}=w+W \mathbf{x}_{n}
$$

is a diffeomorphism of $\mathbb{R}_{+}^{n}$ onto $\Omega$, while the inverse diffeomorphism $\mathfrak{g}=g^{-1}$ is represented by the formula

$$
\mathfrak{g}(y)=\left(y^{\prime}, \mathfrak{G}(y)\right)
$$

with a Lipschitz function $\mathfrak{G} \in C^{\infty}(\Omega)$ satisfying the inequalities

$$
\begin{aligned}
& \left\|\left(D^{\alpha} \mathfrak{G}\right) \circ g\right\|_{L^{\infty}\left(I^{\bigotimes}\right)} \leqslant c(\alpha, \theta) l_{I}^{1-|\alpha|} b_{I}, \quad|\alpha|>1, \\
& \left\|\left(D^{\alpha} \mathfrak{G}\right) \circ g\right\|_{L^{\infty}\left(I^{\bigotimes}\right)} \leqslant c(\alpha, \theta) l_{I}^{1-|\alpha|}, \quad \alpha \neq 0 .
\end{aligned}
$$

The operator

$$
A=\sum_{i=1}^{n-1}\left\{D_{i i}+\frac{\partial \mathfrak{G}}{\partial y_{i}}(g) D_{i n}+\frac{\partial \mathfrak{G}}{\partial y_{i}}(g) D_{n i}\right\}+|\nabla \mathfrak{G}(g)|^{2} D_{n n}
$$

belongs to $\mathcal{A}_{\lambda}^{\mu}$ for some $\lambda(n, \theta) \geqslant 1$ and each $0<\mu<1$. The inequalities

$$
\begin{aligned}
& |A|_{I} \leqslant c(n, \theta) b_{I}, \\
& \|L\|_{I} \leqslant c(n, \theta) l_{I}^{-1} b_{I} \quad \text { for } L=-(\Delta \mathfrak{G}) \circ g=-\sum_{i=1}^{n}\left(D_{i i} \mathfrak{G}\right) \circ g
\end{aligned}
$$

hold true.

We call $\left(\left\{\gamma_{K}\right\}, w, W, g, \mathfrak{g}, \mathfrak{G}, A, \lambda, L\right)$ the standard set of the pair $(\omega, \theta)$.

Proof. It is obvious that $w \in C^{\infty}\left(\mathbb{R}_{+}^{n}\right)$. It is elementary to check (it is sufficient to consider one-dimensional dyadic intervals) that

$$
\text { if }\left(I^{\times}\right)^{\circ} \cap \overline{\frac{3}{2} K} \neq \varnothing \text { and } l_{K} \in\left\{l_{I} / 2, l_{I}, 2 l_{I}\right\} \text {, then } K \subset 5 I \text {. }
$$

By analogy with [10, Subsect. 2.7], now one can obtain properties 42-46) and the estimate

$$
\left\|\omega-\gamma_{I}\right\|_{L^{\infty}(5 I)} \leqslant c_{1}(n, \theta) l_{I}, \quad I \in \mathcal{D} .
$$

The Lipschitz property for $w$ is implied by inequalities (46) with $|\alpha|=1$.

By (7a) and (7b), the function $w$ coincides with the polynomial $\gamma_{I} \circ \mathbf{x}^{\prime}$ in some neighbourhood of the point $\left(\mathfrak{c}_{I}, 11 l_{I} / 8\right) \in I^{\unrhd}$. By (45), the Taylor formula, the convexity of the parallelepiped $I^{\otimes}$ and (44) we obtain

$$
\begin{aligned}
& \left\|w-\gamma_{I} \circ \mathbf{x}^{\prime}\right\|_{L^{\infty}\left(I^{\boxplus}\right)} \leqslant c(n) l_{I} b_{I}, \\
& \left\|w-\gamma_{I} \circ \mathbf{x}^{\prime}\right\|_{L^{\infty}\left(I^{\boxplus}\right)} \leqslant c_{2}(n, \theta) l_{I} .
\end{aligned}
$$


It follows immediately from $(46)$ that $\left\|D_{n} w\right\|_{L^{\infty}\left(\mathbb{R}_{+}^{n}\right)} \leqslant c_{3}(n, \theta)$. We let

$$
W(n, \theta)=3 \max \left\{c_{1}, c_{2}, c_{3}\right\} .
$$

Inequalities (47) and (48) are trivial. The required properties of the mappings $g$ and $\mathfrak{g}$ including estimates (49) and (50) on $\mathfrak{G}$ are obtained due to Theorem 2.5 in [10].

The bi-Lipschitz constant of the mapping $g$ is less than some number $c(n, \theta)$ due to (46) and 50 . Hence, for some $\lambda(n, \theta) \geqslant 1$, one can easily obtain the uniform ellipticity condition $A[x] \in \mathcal{A}_{\lambda}$, see [9]. Hence, $A \in \mathcal{A}_{\lambda}^{\mu}$.

By inequalities (46), (49) and (50) we have

$$
\left\|D a_{i j}\right\|_{L^{\infty}\left(I^{\boxplus}\right)}+\|L\|_{L^{\infty}\left(I^{\boxplus}\right)}+l_{I}\|D L\|_{L^{\infty}\left(I^{\boxplus}\right)} \leqslant c(n, \theta) l_{I}^{-1} b_{I} .
$$

Now (51) and (52) are obtained by the analogue of estimate (2) for the set $I^{凶}$.

Remark. The operator $A$ and function $L$ are such that each function $U$ harmonic in the domain $\Omega$ solves the equation $A(U \circ g)=L D_{n}(U \circ g)$.

In the rest of the section we restrict ourselves by the functions $\omega \in \operatorname{LIP}$.

Definition 1. The set LIP consists of Lipschitz functions

$$
\mathbb{R}^{n-1} \rightarrow \mathbb{R}
$$

each of which coincides with some polynomial in $\mathbb{P}_{1}^{n-1}$ on the complement of some compact set.

Let us find out, what Theorem 2 gives once it is applied to the potential $\Phi_{A w}$.

Lemma 4. Let $\omega \in \operatorname{LIP}$ and $I \in \mathcal{D}$. Then

$$
\Theta_{1}:=\sum_{J} \Gamma_{I J}^{(0, n)} b_{J}<\infty, \quad \Theta_{2}:=\sum_{J} \Gamma_{I J}^{(0, n)} b_{J}^{2}<\infty, \quad \Theta_{2}^{*}:=\sum_{J} \Gamma_{I J}^{(1, n)} b_{J}^{2}<\infty .
$$

Given a constant $\theta \geqslant\|\omega\|_{\text {Lip }}$, let $\left(\left\{\gamma_{K}\right\}, w, W, g, \mathfrak{g}, \mathfrak{G}, A, \lambda, L\right)$ be a standard set of the pair $(\omega, \theta)$. As $k \geqslant 0$, we denote

Then the inequalities

$$
\gamma_{k}^{\prime}=\gamma_{I^{(k)}}, \quad \tau_{1, k}=D_{1} \gamma_{k}^{\prime}, \quad \ldots, \quad \tau_{n-1, k}=D_{n-1} \gamma_{k}^{\prime} .
$$

$$
\begin{aligned}
& \left\|\omega-\gamma_{k+1}^{\prime}\right\|_{L^{2}\left(5 I^{(k)}\right)}+\left\|\omega-\gamma_{k}^{\prime}\right\|_{L^{2}\left(5 I^{(k)}\right)} \leqslant c(n) l_{I^{(k)}}^{\frac{n+1}{2}} b_{I^{(k)}}, \\
& l_{I^{(k)}}^{-1}\left\|\gamma_{k+1}^{\prime}-\gamma_{k}^{\prime}\right\|_{L^{\infty}\left(5 I^{(k)}\right)}+\left|\nabla\left(\gamma_{k+1}^{\prime}-\gamma_{k}^{\prime}\right)\right| \leqslant c(n) b_{I^{(k)}}
\end{aligned}
$$

hold true.

For each $\mu \in(0,1)$ and the function $f=w$ all assumptions of Theorem 2 hold and in terms of the notations of this theorem the relations

$$
\begin{aligned}
& \Theta \leqslant c(n, \theta) \Theta_{2}, \\
& \Theta^{*} \leqslant c(n, \theta) l_{I}^{-1} \Theta_{2}^{*}, \\
& A_{k}=\sum_{i=1}^{n-1}\left\{D_{i i}-\frac{\tau_{i, k}}{W} D_{i n}-\frac{\tau_{i, k}}{W} D_{n i}\right\}+\frac{1+\sum_{s=1}^{n-1} \tau_{s, k}^{2}}{W^{2}} D_{n n}, \\
& \gamma_{k}(x)=\gamma_{k}^{\prime}\left(x^{\prime}\right), \\
& F_{k}(x)=W \frac{\Gamma(n / 2)}{\pi^{n / 2}} \lim _{r \rightarrow \infty} \int_{\left|\xi-\mathfrak{c}_{k}^{\prime}\right|<r} \frac{\omega_{(k)}(\xi)}{\left|\left(x^{\prime}, \gamma_{k}^{\prime}\left(x^{\prime}\right)+W x_{n}\right)-\left(\xi, \gamma_{k}^{\prime}(\xi)\right)\right|^{n}} d \xi
\end{aligned}
$$

hold, where

$$
\omega_{(k)}=\omega_{k+1}-\omega_{k}, \quad \omega_{k}=\varphi_{k}^{\prime} \omega+\left(1-\varphi_{k}^{\prime}\right) \gamma_{k}^{\prime}, \quad \varphi_{k}^{\prime}(\xi)=\varphi_{k}(\xi, 0+) .
$$

Remark. The limit $\varphi_{k}^{\prime}(\xi)$ exists thanks to inequality 4c. 
Proof. Due to (44) we have $b_{J} \leqslant c_{1}(n, \theta)$. The definition of the set LIP and the definition of the numbers $b_{J}$ show that

$$
b_{J} \leqslant C_{1}(\omega) l_{J}^{-\frac{n+1}{2}} \leqslant C_{2}\left(\omega, l_{I}\right) l_{I}^{\frac{n+1}{2}} l_{J}^{-\frac{n+1}{2}}
$$

that by Lemma 1 yields

$$
b_{J} \leqslant \min \left\{c_{1}, C_{2} l_{I}^{\frac{n+1}{2}} l_{J}^{-\frac{n+1}{2}}\right\} \leqslant c_{1}^{\frac{n}{n+1}} C_{2}^{\frac{1}{n+1}} l_{I}^{1 / 2} l_{J}^{-1 / 2}, \quad \Theta_{1} \leqslant c_{1}^{\frac{n}{n+1}} C_{2}^{\frac{1}{n+1}} \sum_{J} \Gamma_{I J}^{(1 / 2, n-1 / 2)}<\infty .
$$

The relations $\Theta_{2}<\infty$ and $\Theta_{2}^{*}<\infty$ are implied by the inequalities $b_{J} \leqslant c_{1}$ and $\Theta_{1}<\infty$.

Estimates (54) and (55) are obtained by the embeddings $I^{(k)} \subset I^{(k+1)} \subset 5 I^{(k)}$ and simple properties of the polynomials similarly to [10, Subsect. 2.7].

The conditions $\lambda \geqslant 1$ and $A \in \mathcal{A}_{\lambda}^{\mu}$ of Theorem 2 are implied by Theorem 3 . In view of (2) and 45 we have

$$
\begin{aligned}
& \left\|D^{2} w\right\|_{J} \leqslant c_{2}(n) l_{J}^{-1} b_{J}, \\
& \sum_{J} \Gamma_{I J}^{(0, n)} l_{J}\left\|D^{2} w\right\|_{J} \leqslant c_{2} \Theta_{1}<\infty,
\end{aligned}
$$

and hence, $D^{2} w \in \mathrm{VL}(0)$. By (51), (61) and the Cauchy inequality we get

$$
\begin{aligned}
& \mathcal{F}_{J} \leqslant c_{2} l_{J}^{-1} b_{J}+c_{2}[I, J]^{-1} \sum_{H \in\left\{I^{J}\right\} \cup \overrightarrow{I J}} b_{H} \leqslant 2 c_{2} l_{J}^{-1} \sum_{H \in \overleftarrow{I J} \cup \overrightarrow{I J}} b_{H}, \\
& \left(\sum_{H \in \overleftarrow{I J} \cup \overrightarrow{I J}}|A|_{H}\right) \mathcal{F}_{J} \leqslant c(n, \theta) l_{J}^{-1}\left(\sum_{H \in \overleftarrow{I J} \cup \overrightarrow{I J}} b_{H}\right)^{2} \leqslant c_{3}(n, \theta) l_{J}^{-3 / 2} \sum_{H \in \overleftarrow{I J} \cup \overrightarrow{I J}} l_{H}^{1 / 2} b_{H}^{2} .
\end{aligned}
$$

By (32) we obtain

$$
\sum_{J: H \in \overleftarrow{I J} \cup \overrightarrow{I J}} \Gamma_{I J}^{(0, n)} l_{J} l_{J}^{-3 / 2} \leqslant c_{4}(n) \Gamma_{I H}^{(0, n)} l_{H}^{-1 / 2}, \quad \sum_{J: H \in \overleftarrow{I J} \cup \overrightarrow{I J}} \Gamma_{I J}^{(0, n+1)} l_{J}^{-3 / 2} \leqslant c_{5}(n) l_{I}^{-1} \Gamma_{I H}^{(1, n)} l_{H}^{-1 / 2}
$$

Therefore,

$$
\begin{aligned}
& \Theta \leqslant c_{3} \sum_{H}\left(\sum_{J: H \in \overleftarrow{I J} \cup \overrightarrow{I J}} \Gamma_{I J}^{(0, n)} l_{J} l_{J}^{-3 / 2}\right) l_{H}^{1 / 2} b_{H}^{2} \leqslant c_{3} c_{4} \Theta_{2}, \\
& \Theta^{*} \leqslant c_{3} \sum_{H}\left(\sum_{J: H \in \overleftarrow{I J} \cup \overrightarrow{I J}} \Gamma_{I J}^{(0, n+1)} l_{J}^{-3 / 2}\right) l_{H}^{1 / 2} b_{H}^{2} \leqslant c_{3} c_{5} l_{I}^{-1} \Theta_{2}^{*} .
\end{aligned}
$$

We have obtained estimates (56) and (57), which imply $\Theta<\infty$. Hence, the function $f=w$ satisfies all assumptions of Theorem 2 .

By (7a) the function $w(x)$ coincides with $\gamma_{k}^{\prime}\left(x^{\prime}\right)$ in a "half-neighbourhood" of the point $\mathfrak{c}_{k}$, while the function $\mathfrak{G}(y)$ coincides with the function $\frac{y_{n}-\gamma_{k}^{\prime}\left(y^{\prime}\right)}{W}$ in a "half-neighbourhood" of the point $g\left(\mathfrak{c}_{k}\right)$. This leads us to (58) and (59).

Let us prove identity (60). In view of (58) it is easy to confirm that

$$
\operatorname{det}_{A_{k}}=W^{-2} \text {. }
$$

We omit the subscript $k$ in notation of the numbers $\tau_{i, k}$ and the coefficients $a_{i j, k}$ of the operator $A_{k}$. Introducing the shorthand notation $\tau_{n}=W$, we can write (58) as

$$
a_{i j}=\delta_{i j}-\delta_{i n} \frac{\tau_{j}}{W}-\delta_{j n} \frac{\tau_{i}}{W}+\delta_{i n} \delta_{j n} \frac{1+\sum_{s=1}^{n} \tau_{s}^{2}}{W^{2}} .
$$


The numbers

$$
b_{i j}=\delta_{i j}-\delta_{i n} \delta_{j n}+\tau_{i} \tau_{j}
$$

satisfy the identities

$$
\begin{aligned}
\sum_{j=1}^{n} a_{i j} b_{j q}= & \sum_{j} a_{i j}\left(\delta_{j q}-\delta_{j n} \delta_{q n}+\tau_{j} \tau_{q}\right)=a_{i q}-a_{i n} \delta_{q n}+\left(\sum_{j} a_{i j} \tau_{j}\right) \tau_{q} \\
a_{i q}-a_{i n} \delta_{q n}= & \delta_{i q}-\delta_{i n} \frac{\tau_{q}}{W}-\delta_{q n} \frac{\tau_{i}}{W}+\delta_{i n} \delta_{q n} \frac{1+\sum_{s=1}^{n} \tau_{s}^{2}}{W^{2}} \\
& -\left(\delta_{i n}-\delta_{i n}-\frac{\tau_{i}}{W}+\delta_{i n} \frac{1+\sum_{s=1}^{n} \tau_{s}^{2}}{W^{2}}\right) \delta_{q n}=\delta_{i q}-\delta_{i n} \frac{\tau_{q}}{W} \\
\sum_{j} a_{i j} \tau_{j}= & \tau_{i}-\frac{\delta_{i n}}{W} \sum_{j} \tau_{j}^{2}+\left(-\frac{\tau_{i}}{W}+\delta_{i n} \frac{1+\sum_{s=1}^{n} \tau_{s}^{2}}{W^{2}}\right) \tau_{n}=\frac{\delta_{i n}}{W} \\
\sum_{j=1}^{n} a_{i j} b_{j q}= & \delta_{i q}-\delta_{i n} \frac{\tau_{q}}{W}+\frac{\delta_{i n}}{W} \tau_{q}=\delta_{i q}
\end{aligned}
$$

Hence, $\left(b_{i j}\right)=\left(a_{i j}\right)^{-1}$. Letting $\xi_{n}=0$, for $\xi \in \mathbb{R}^{n-1}$ we have

$$
\begin{aligned}
Q_{A_{k}}(x-(\xi, 0)) & =\sum_{i, j=1}^{n} b_{i j}\left(x_{i}-\xi_{i}\right)\left(x_{j}-\xi_{j}\right)=\sum_{i=1}^{n-1}\left(x_{i}-\xi_{i}\right)^{2}+\sum_{i, j=1}^{n} \tau_{i} \tau_{j}\left(x_{i}-\xi_{i}\right)\left(x_{j}-\xi_{j}\right) \\
& =\left|x^{\prime}-\xi\right|^{2}+\left(\gamma_{k}^{\prime}\left(x^{\prime}\right)-\gamma_{k}^{\prime}(\xi)+\tau_{n} x_{n}-\tau_{n} \xi_{n}\right)^{2} \\
& =\left|\left(x^{\prime}, \gamma_{k}^{\prime}\left(x^{\prime}\right)+W x_{n}\right)-\left(\xi, \gamma_{k}^{\prime}(\xi)\right)\right|^{2}
\end{aligned}
$$

which by 42 and $(59)$ leads us to 60 . The proof is complete.

Under the assumptions of Lemma 4 we denote

$$
H_{0}=\left\{x \in \bar{I} \times \mathbb{R}: x_{n} \geqslant \gamma_{0}^{\prime}\left(x^{\prime}\right)+2 W l_{I} / 3\right\}, \quad H_{k}=\bar{I} \times \mathbb{R} \quad \text { as } k \geqslant 1 .
$$

We let $\mathfrak{x}=\mathfrak{c}_{I^{(k)}}$. It is obvious that for $x \in H_{k}$ there exists the limit

$$
F_{(k)}(x)=\frac{\Gamma(n / 2)}{\pi^{n / 2}} \lim _{r \rightarrow \infty} \int_{|\xi-\mathfrak{x}|<r} \omega_{(k)}(\xi)\left|x-\left(\xi, \gamma_{k}^{\prime}(\xi)\right)\right|^{-n} d \xi
$$

We have $g\left(I^{\square}\right) \subset H_{k}$ (in view of $(48)$ ) and $\left(x^{\prime}, \gamma_{k}^{\prime}\left(x^{\prime}\right)+W x_{n}\right) \in H_{k}$ for $x \in I^{\square}$, see 60 .

Lemma 5. Under assumptions of Lemma 4 for $(x, \xi) \in H_{k} \times \mathbb{R}^{n-1}$ we let

$$
\xi^{*}=2 \mathfrak{x}-\xi, \quad M_{k}(x, \xi)=\frac{1}{2}\left\{\omega_{(k)}(\xi)\left|x-\left(\xi, \gamma_{k}^{\prime}(\xi)\right)\right|^{-n}+\omega_{(k)}\left(\xi^{*}\right)\left|x-\left(\xi^{*}, \gamma_{k}^{\prime}\left(\xi^{*}\right)\right)\right|^{-n}\right\} .
$$

Then $F_{(k)} \in C^{\infty}\left(H_{k}\right)$ and for each $\alpha \in \mathbb{N}_{0}^{n}$

$$
\begin{gathered}
\int_{\mathbb{R}^{n-1}}\left|D_{x}^{\alpha} M_{k}(x, \xi)\right| d \xi \leqslant c(\alpha, \theta) l_{I^{(k)}}^{-|\alpha|} b_{I^{(k)}}, \\
D^{\alpha} F_{(k)}(x)=\frac{\Gamma(n / 2)}{\pi^{n / 2}} \int_{\mathbb{R}^{n-1}} D_{x}^{\alpha} M_{k}(x, \xi) d \xi .
\end{gathered}
$$

Proof. We denote

$$
\mathfrak{X}=\left(\mathfrak{x}, \gamma_{k}^{\prime}(\mathfrak{x})\right), \quad \Xi=|x-\mathfrak{X}|+|\mathfrak{x}-\xi| .
$$

Let $T_{k}(\xi)$ be the convex hull of the set $\left\{\gamma_{k}^{\prime}(\xi), \omega(\xi), \omega_{k+1}(\xi)\right\} \subset \mathbb{R}$. 
Let us prove that

$$
\begin{aligned}
& \text { if }(x, \xi) \in H_{k} \times \mathbb{R}^{n-1} \text { with } t \in T_{k}(\xi), \text { and } \xi \notin 3 I^{(k-1)} \text { for } k \geqslant 1, \\
& \text { then }|x-(\xi, t)| \geqslant c(n, \theta) \Xi \geqslant c(n, \theta) l_{I^{(k)}} .
\end{aligned}
$$

If the assumption in 65 holds, then $t=\zeta(\xi)$ for a convex linear combination

$$
\zeta=\beta_{1} \gamma_{k}^{\prime}+\beta_{2} \omega+\beta_{3} \gamma_{k+1}^{\prime},
$$

since $\omega_{k+1}=\varphi_{k+1}^{\prime} \omega+\left(1-\varphi_{k+1}^{\prime}\right) \gamma_{k+1}^{\prime}$. We denote

$$
Z=\left(x^{\prime}, \zeta\left(x^{\prime}\right)\right), \quad R=|x-Z|+\left|x^{\prime}-\xi\right| .
$$

It follows from the inequalities $\theta \geqslant\|\omega\|_{\text {Lip }}$ and (43) that $\|\zeta\|_{\text {Lip }} \leqslant c(n) \theta$ and this is why by the triangle with the vertices $x, Z$ and $(\xi, t)=(\xi, \zeta(\xi))$ we get that

$$
|x-(\xi, t)| \geqslant c_{1}(n, \theta)\{|x-Z|+|Z-(\xi, t)|\} \geqslant c_{1} R .
$$

Let us treat the cases $\xi \in 3 I$ and $\xi \notin 3 I$. If $\xi \in 3 I$, then $k=0$ and $\omega_{k+1}(\xi)=\omega(\xi)$ due to (4a) and hence, $\beta_{3}=0$ without loss of generality. By $x \in H_{0}$ and (47) we have

$$
\begin{aligned}
& x_{n}-\gamma_{0}^{\prime}\left(x^{\prime}\right) \geqslant 2 W l_{I} / 3, \\
& x_{n}-\omega\left(x^{\prime}\right) \geqslant x_{n}-\gamma_{0}^{\prime}\left(x^{\prime}\right)-W l_{I} / 3 \geqslant W l_{I} / 3, \\
& R \geqslant|x-Z|=\beta_{1}\left[x_{n}-\gamma_{0}^{\prime}\left(x^{\prime}\right)\right]+\beta_{2}\left[x_{n}-\omega\left(x^{\prime}\right)\right] \geqslant W l_{I} / 3 .
\end{aligned}
$$

If $\xi \notin 3 I$, then $\left|x^{\prime}-\xi\right| \geqslant l_{I}$ as $k=0$ and $\left|x^{\prime}-\xi\right| \geqslant l_{I^{(k-1)}}$ as $k \geqslant 1$ and hence,

$$
R \geqslant \min \{W / 3,1 / 2\} l_{I^{(k)}} \text { for each } \xi .
$$

By (43), 44), 47) and (55) we conclude that

$$
\begin{aligned}
& \left|\omega\left(x^{\prime}\right)-\gamma_{k}^{\prime}\left(x^{\prime}\right)\right| \leqslant\left\|\omega-\gamma_{I^{(k)}}\right\|_{L^{\infty}\left(I^{(k)}\right)} \leqslant W l_{I^{(k)}} / 3, \quad\left|\gamma_{k+1}^{\prime}\left(x^{\prime}\right)-\gamma_{k}^{\prime}\left(x^{\prime}\right)\right| \leqslant c(n, \theta) l_{I^{(k)}}, \\
& |\mathfrak{X}-Z| \leqslant\left|\left(\mathfrak{x}, \gamma_{k}^{\prime}(\mathfrak{x})\right)-\left(x^{\prime}, \gamma_{k}^{\prime}\left(x^{\prime}\right)\right)\right|+\left|\gamma_{k}^{\prime}\left(x^{\prime}\right)-\zeta\left(x^{\prime}\right)\right| \leqslant c(n, \theta) l_{I^{(k)}}, \\
& \Xi \leqslant R+|\mathfrak{X}-Z|+\left|\mathfrak{x}-x^{\prime}\right| \leqslant R+c(n, \theta) l_{I^{(k)}} \leqslant c_{2}(n, \theta) R, \quad|x-(\xi, t)| \geqslant c_{1} c_{2}^{-1} \Xi .
\end{aligned}
$$

If $\xi \in 3 I$, then $k=0$ and $\gamma_{0}^{\prime}(\mathfrak{x}) \in T_{0}(\mathfrak{x})$ and therefore,

$$
\Xi \geqslant|x-\mathfrak{X}| \geqslant\left. c_{1} R\right|_{\xi=\mathfrak{x}} \geqslant c_{1} \min \{W / 3,1\} l_{I} .
$$

If $\xi \notin 3 I$, then $|\mathfrak{x}-\xi| \geqslant 3 l_{I} / 2$ as $k=0,|\mathfrak{x}-\xi| \geqslant l_{I^{(k-1)}}$ as $k \geqslant 1$ and hence, $\Xi \geqslant l_{I^{(k)}} / 2$ for each $k$. Thus, $\Xi \geqslant c(n, \theta) l_{I^{(k)}}$ for each $\xi$ and we complete the proof of implication (65).

If $\omega_{(k)}(\xi) \neq 0$, then $\xi \notin 3 I^{(k-1)}$ as $k \geqslant 1$ due to (4a) and hence, by (65),

$$
\begin{aligned}
& \text { if }(x, \xi) \in H_{k} \times \mathbb{R}^{n-1} \text { and either } \omega_{(k)}(\xi) \neq 0 \text {, or } \xi \notin 5 I^{(k)}, \\
& \text { then }\left|D_{x}^{\alpha}\right| x-\left.(\xi, t)\right|^{-n} \mid \leqslant c(\alpha, \theta) \Xi^{-n-|\alpha|} \leqslant c(\alpha, \theta) l_{I^{(k)}}^{-n-|\alpha|} \text { as } t \in T_{k}(\xi) .
\end{aligned}
$$

By (54) and the Hölder inequality this follows that

$$
\begin{aligned}
& \omega_{(k)}=\left(\varphi_{k+1}^{\prime}-1\right)\left(\omega-\gamma_{k+1}^{\prime}\right)+\left(1-\varphi_{k}^{\prime}\right)\left(\omega-\gamma_{k}^{\prime}\right) \\
& \left\|\omega_{(k)}\right\|_{L^{1}\left(5 I^{(k)}\right)} \leqslant c(n) l_{I^{(k)}}^{n} b_{I^{(k)}} \\
& \left\|D_{x}^{\alpha} M_{k}(x, \cdot)\right\|_{L^{1}\left(5 I^{(k)}\right)} \leqslant c(\alpha, \theta)\left\|\omega_{(k)}\right\|_{L^{1}\left(5 I^{(k)}\right)} l_{I^{(k)}}^{-n-|\alpha|} \leqslant c(\alpha, \theta) l_{I^{(k)}}^{-|\alpha|} b_{I^{(k)}} .
\end{aligned}
$$


Let $\xi \in \mathbb{R}^{n-1} \backslash \overline{5 I^{(k)}}\left(\Rightarrow \xi^{*} \in \mathbb{R}^{n-1} \backslash 5 I^{(k)}\right)$. Then $\omega_{(k)}(\xi)=\gamma_{k+1}^{\prime}(\xi)-\gamma_{k}^{\prime}(\xi)$ and $\omega_{(k)}\left(\xi^{*}\right)=$ $\gamma_{k+1}^{\prime}\left(\xi^{*}\right)-\gamma_{k}^{\prime}\left(\xi^{*}\right)$ due to (4b). By (55) and (66) we obtain

$$
\begin{aligned}
& \left|\frac{\omega_{(k)}(\xi)+\omega_{(k)}\left(\xi^{*}\right)}{2}\right|=\left|\gamma_{k+1}^{\prime}(\mathfrak{x})-\gamma_{k}^{\prime}(\mathfrak{x})\right| \leqslant c(n) l_{I^{(k)}} b_{I^{(k)}}, \\
& \left|\frac{\omega_{(k)}(\xi)+\omega_{(k)}\left(\xi^{*}\right)}{2} D_{x}^{\alpha}\right| x-\left.\left(\xi, \gamma_{k}^{\prime}(\xi)\right)\right|^{-n} \mid \leqslant c(\alpha, \theta) l_{I^{(k)}} b_{I^{(k)}} \Xi^{-n-|\alpha|}, \\
& \left|\omega_{(k)}\left(\xi^{*}\right)\right| \leqslant c(n)\left(l_{I^{(k)}}+\left|\mathfrak{x}-\xi^{*}\right|\right) b_{I^{(k)}} \leqslant c(n)|\mathfrak{x}-\xi| b_{I^{(k)}} \leqslant c(n) \Xi b_{I^{(k)}}, \\
& \left|D_{x}^{\alpha} M_{k}(x, \xi)\right| \leqslant c(\alpha, \theta) l_{I^{(k)}} b_{I^{(k)}} \Xi^{-n-|\alpha|}+c(n) \Xi b_{I^{(k)}}\left|Y_{\alpha}\right|,
\end{aligned}
$$

where

$$
Y_{\alpha}=D_{x}^{\alpha}\left|x-\left(\xi, \gamma_{k}^{\prime}(\xi)\right)\right|^{-n}-D_{x}^{\alpha}\left|x-\left(\xi^{*}, \gamma_{k}^{\prime}\left(\xi^{*}\right)\right)\right|^{-n}
$$

Majorizing each term by 66 , we get

$$
\begin{aligned}
\left|Y_{\alpha}\right| \leqslant c(\alpha, \theta) & \Xi^{-n-|\alpha|} \\
\left|D_{x}^{\alpha} M_{k}(x, \xi)\right| & \leqslant c_{3}(\alpha, \theta)\left(l_{I^{(k)}}+\Xi\right) b_{I^{(k)}} \Xi^{-n-|\alpha|} \\
& \leqslant \frac{7 c_{3}}{5} b_{I^{(k)}} \Xi^{1-n-|\alpha|} \leqslant \frac{7 c_{3}}{5} b_{I^{(k)}}|\mathfrak{x}-\xi|^{1-n-|\alpha|} .
\end{aligned}
$$

Relations $x^{\prime} \in \bar{I} \subset \overline{I^{(k)}}, \mathfrak{X}^{\prime}=\mathfrak{x} \in I^{(k)}$ and $\xi \notin 5 I^{(k)}$ show that

$$
\begin{aligned}
& \left|\tau x^{\prime}+(1-\tau) \mathfrak{x}-\xi\right|_{\infty} \geqslant \frac{4}{5}|\mathfrak{x}-\xi|_{\infty}, \\
& |\tau x+(1-\tau) \mathfrak{X}-(\xi, t)| \geqslant \frac{4}{5 \sqrt{n-1}}|\mathfrak{x}-\xi| \quad \text { for each } \tau \in[0,1] \text { and } t \in \mathbb{R} .
\end{aligned}
$$

Hence, by the identity $\left|\mathfrak{X}-\left(\xi, \gamma_{k}^{\prime}(\xi)\right)\right|=\left|\mathfrak{X}-\left(\xi^{*}, \gamma_{k}^{\prime}\left(\xi^{*}\right)\right)\right|$ and by (71) we obtain

$$
\begin{aligned}
& \left|Y_{0}\right| \leqslant c(n)|x-\mathfrak{X}||\mathfrak{x}-\xi|^{-n-1}, \\
& \left|Y_{0}\right| \leqslant \min \left\{c(n)|x-\mathfrak{X}||\mathfrak{x}-\xi|^{-n-1}, c(n, \theta) \Xi^{-n}\right\} \leqslant c(n, \theta)|x-\mathfrak{X}| \Xi^{-n-1}, \\
& \left|M_{k}(x, \xi)\right| \leqslant c(n, \theta)\left(l_{I^{(k)}}+|x-\mathfrak{X}|\right) b_{I^{(k)}} \Xi^{-n}, \\
& \int_{|\mathfrak{x}-\xi| \geqslant 5 l_{I^{(k)} / 2}}(|x-\mathfrak{X}|+|\mathfrak{x}-\xi|)^{-n} d \xi \leqslant c(n)\left(l_{I^{(k)}}+|x-\mathfrak{X}|\right)^{-1}, \\
& \left\|M_{k}(x, \cdot)\right\|_{L^{1}\left(\mathbb{R}^{n-1} \backslash 5 I^{(k)}\right)} \leqslant c(n, \theta) b_{I^{(k)}} .
\end{aligned}
$$

Estimate (64a) as $\alpha=0$ is implied by 68 and $(74)$, while as $\alpha \neq 0$, it is due to (68) and (72). Identity (64b) as $\alpha=0$ is yielded by (63) and the change of variable $\xi \rightarrow \xi^{*}$, while as $\alpha \neq 0$ (together with the statement $F_{(k)} \in C^{\infty}\left(H_{k}\right)$ ) it is implied by differentiating the integral formula $64 \mathrm{~b}$ ) that is possible thanks to 72 .

3.2. Function $S$ and potential $\Phi_{L}$. Let us give a "qualitative" analogue of Lemma 5 for the functions determined by the volume integrals.

Lemma 6. Let $\omega_{+}, \omega_{-} \in \operatorname{LIP}, \Omega_{ \pm}=\left\{x \in \mathbb{R}^{n}: x_{n}>\omega_{ \pm}\left(x^{\prime}\right)\right\}$ and $\chi=\chi_{+}-\chi_{-}$, where $\chi_{ \pm}$ are the characteristic functions of the sets $\Omega_{+}$and $\Omega_{-}$. We let

$$
\xi^{*}=2 \mathfrak{x}-\xi, \quad N_{\mathfrak{x}}(x, \xi)=\int_{\mathbb{R}} \frac{\chi(\xi, t)|x-(\xi, t)|^{-n}+\chi\left(\xi^{*}, t\right)\left|x-\left(\xi^{*}, t\right)\right|^{-n}}{2} d t
$$

for $(\mathfrak{x}, x, \xi) \in \mathbb{R}^{n-1} \times\left(\mathbb{R}^{n} \backslash \operatorname{supp} \chi\right) \times \mathbb{R}^{n-1}$. Then the following statements hold true. 
(i) The function $N_{x^{\prime}}(x, \cdot)$ belongs to $L^{1}\left(\mathbb{R}^{n-1}\right)$, there exists the limit

$$
s(x)=\lim _{r \rightarrow \infty} \int_{|x-y|<r} \chi(y)|x-y|^{-n} d y,
$$

and the identity $s(x)=\int_{\mathbb{R}^{n-1}} N_{x^{\prime}}(x, \xi) d \xi$ holds true.

(ii) For each $(\mathfrak{x}, x, \alpha) \in \mathbb{R}^{n-1} \times\left(\mathbb{R}^{n} \backslash \operatorname{supp} \chi\right) \times \mathbb{N}_{0}^{n}$ the belonging

$$
D_{x}^{\alpha} N_{\mathfrak{x}}(x, \cdot) \in L^{1}\left(\mathbb{R}^{n-1}\right)
$$

holds true, the function $s$ is infinitely differentiable in $\mathbb{R}^{n} \backslash \operatorname{supp} \chi$ and

$$
D^{\alpha} s(x)=\int_{\mathbb{R}^{n-1}} D_{x}^{\alpha} N_{\mathfrak{x}}(x, \xi) d \xi .
$$

Proof. (i) For $\omega \in \mathrm{LIP}$ by $\chi[\omega]$ we denote the characteristic function of the overgraph of the function $\omega$, while by $\gamma[\omega]$ we denote the polynomial in $\mathbb{P}_{1}^{n-1}$, with which $\omega$ coincides in the vicinity of infinity. As $x \notin \operatorname{supp} \chi$, we let

$$
\gamma_{ \pm}=\gamma\left[\omega_{ \pm}\right] \quad \& \quad \gamma^{ \pm}=\gamma_{ \pm}-\gamma_{ \pm}\left(x^{\prime}\right)+x_{n}
$$

The functions $\chi_{+}$and $\chi_{-}$coincide in the vicinity of $x$ and this is why there exist $\omega^{ \pm} \in \operatorname{LIP}$ such that

$$
\chi_{+}=\chi\left[\omega^{+}\right]=\chi\left[\omega^{-}\right]=\chi_{-} \text {in the vicinity of } x \quad \& \quad \gamma^{ \pm}=\gamma\left[\omega^{ \pm}\right] .
$$

In view of the representation

$$
\chi=\left\{\chi_{+}-\chi\left[\omega^{+}\right]\right\}+\left\{\chi\left[\omega^{+}\right]-\chi\left[\omega^{-}\right]\right\}+\left\{\chi\left[\omega^{-}\right]-\chi_{-}\right\}
$$

we see that in order to check Statement (i), it is sufficient to check (i) for the pairs $\left(\omega_{+}, \omega^{+}\right)$, $\left(\omega^{+}, \omega^{-}\right)$and $\left(\omega^{-}, \omega_{-}\right)$instead of $\left(\omega_{+}, \omega_{-}\right)$. Therefore, it is sufficient to check (i) in particular cases

(a) $\gamma_{+}-\gamma_{-}=$const;

(b) $\gamma_{+}\left(x^{\prime}\right)=x_{n}=\gamma_{-}\left(x^{\prime}\right)$.

In Case (a), the function $y \mapsto \chi(y)|x-y|^{-n}$ belongs to $L^{1}\left(\mathbb{R}^{n}\right)$, which gives (i) by the Fubini theorem and the change of variables $\xi \rightarrow \xi^{\star}=2 x^{\prime}-\xi$. In Case (b), the change of variables $y=(\xi, t) \rightarrow 2 x-y$ and the Fubini theorem shows that

$$
\begin{aligned}
& N_{x^{\prime}}(x, \xi)=0 \quad \text { for large }\left|x^{\prime}-\xi\right|, \\
& \left(\exists r_{0}>0\right)\left(\forall r>r_{0}\right) \quad \int_{|x-y|<r} \chi(y)|x-y|^{-n} d y=\int_{\mathbb{R}^{n-1}} N_{x^{\prime}}(x, \xi) d \xi .
\end{aligned}
$$

Thus, the proof of Statement (i) is complete.

(ii) For $\xi \in \mathbb{R}^{n-1}$ we let

$$
\nu(\xi)=\int_{\mathbb{R}} \frac{\chi\left(\xi^{\star}, t\right)\left|x-\left(\xi^{\star}, t\right)\right|^{-n}-\chi\left(\xi^{*}, t\right)\left|x-\left(\xi^{*}, t\right)\right|^{-n}}{2} d t .
$$

By the identity $\xi^{\star}-\xi^{*}=2 x^{\prime}-2 \mathfrak{x}$ it is easy to get that

$$
\sup _{\xi}|\nu(\xi)||\xi|^{n}<\infty, \quad \nu \in L^{1}\left(\mathbb{R}^{n-1}\right)
$$

and

$$
\int_{\mathbb{R}^{n-1}} \nu(\xi) d \xi=0
$$

This is why properties 75 with $\alpha=0$ are implied by Statement (i). The case $\alpha \neq 0$ can be treated similarly to Lemma 5, via checking an analogue of estimate (72) for the function $N_{\mathfrak{x}}(x, \xi)$. 
Let $\omega \in \mathrm{LIP}$. To compare Lemmata 4, 5and 6, we introduce the function

$$
S(x):=S_{\Omega}(x):=\lim _{r \rightarrow \infty}\left\{\ln r-\frac{\Gamma(n / 2)}{\pi^{n / 2}} \int_{y \in \mathbb{R}^{n} \backslash \Omega:|x-y|<r}|x-y|^{-n} d y\right\}, \quad x \in \Omega .
$$

Here the limit exists since the area of the unit sphere $\mathbb{S}^{n-1} \subset \mathbb{R}^{n}$ is equal to $\frac{2 \pi^{n / 2}}{\Gamma(n / 2)}$. The function $S$ is invariant w.r.t. the shifts and rotations of the domain $\Omega$ in the obvious sense.

Lemma 7. Under assumptions of Lemma 4 let $\Omega_{k}=\left\{x \in \mathbb{R}^{n}: x_{n}>\omega_{k}\left(x^{\prime}\right)\right\}$ and $S_{k}=S_{\Omega_{k}}$. Then the inequalities

$$
\begin{aligned}
& \left\|D^{\alpha} S-D^{\alpha} S_{0}\right\|_{L^{\infty}\left(g\left(I^{\square}\right)\right)} \leqslant c(\alpha, \theta) \sum_{k=0}^{\infty} l_{I^{(k)}}^{-|\alpha|} b_{I^{(k)}}, \quad \alpha \in \mathbb{N}_{0}^{n}, \\
& \left\|D^{\alpha}\left(S \circ g-S_{0} \circ g\right)\right\|_{I} \leqslant c(\alpha, \theta) \sum_{k=0}^{\infty} l_{I^{(k)}}^{-|\alpha|} b_{I^{(k)}}, \quad|\alpha| \leqslant 1, \\
& \left\|D^{\alpha}\left(S \circ g-S_{0} \circ g\right)\right\|_{I} \leqslant c(\alpha, \theta) \sum_{j=0}^{1} l_{I}^{(1-|\alpha|) j} b_{I}^{j} \sum_{k=0}^{\infty} l_{I^{(k)}}^{(j-1)|\alpha|-j} b_{I^{(k)}}, \quad|\alpha| \geqslant 2
\end{aligned}
$$

hold true. If $\varepsilon=0$ as $|\alpha| \leqslant 1$ and $0<\varepsilon \leqslant 2$ as $|\alpha|=2$, then the sum $F$ of the series $\sum_{k=0}^{\infty} F_{k}$ satisfies

$$
\left\|D^{\alpha}\left(F+W S \circ g-W S_{0} \circ g\right)\right\|_{I} \leqslant c(\alpha, \theta, \varepsilon) l_{I}^{-\varepsilon} \sum_{k=0}^{\infty} l_{I^{(k)}}^{\varepsilon-|\alpha|} b_{I^{(k)}}^{2}, \quad|\alpha| \leqslant 2 .
$$

Proof. By the embedding $g\left(I^{\square}\right) \subset H_{k}$ (see 62 ) and by Lemma 5 we have

$$
\left\|D^{\alpha} F_{(k)}\right\|_{L^{\infty}\left(g\left(I^{\square}\right)\right)} \leqslant c(\alpha, \theta) l_{I^{(k)}}^{-|\alpha|} b_{I^{(k)}}, \quad \alpha \in \mathbb{N}_{0}^{n} .
$$

Let us establish the second main inequality

$$
\left\|D^{\alpha} F_{(k)}+D^{\alpha} S_{k+1}-D^{\alpha} S_{k}\right\|_{L^{\infty}\left(g\left(I^{\square}\right)\right)} \leqslant c(\alpha, \theta) l_{I^{(k)}}^{-|\alpha|} b_{I^{(k)}}^{2}, \quad \alpha \in \mathbb{N}_{0}^{n} .
$$

For $x \in g\left(I^{\square}\right) \subset H_{k}$ we let $\mathfrak{x}, F_{(k)}, \xi^{*}, M_{k}, \mathfrak{X}, \Xi$ and $T_{k}$ to have the same meaning as in Lemma 5 and in its proof, while the functions $\chi, N_{\mathfrak{x}}$ and $s$ are defined by Lemma 6 for the pair of the functions $\left(\omega_{+}, \omega_{-}\right)=\left(\omega_{k+1}, \omega_{k}\right)$. We let

$$
\begin{aligned}
& U(\xi)=D_{x}^{\alpha}\left|x-\left(\xi, \gamma_{k}^{\prime}(\xi)\right)\right|^{-n}-D_{x}^{\alpha}\left|x-\left(\xi, \omega_{k}(\xi)\right)\right|^{-n} \\
& V(\xi, \tau)=D_{x}^{\alpha}\left|x-\left(\xi, \omega_{k}(\xi)\right)\right|^{-n}-D_{x}^{\alpha}\left|x-\left(\xi, \omega_{k}(\xi)+\omega_{(k)}(\xi) \tau\right)\right|^{-n} .
\end{aligned}
$$

In view of the belongings $\gamma_{k}^{\prime}(\xi), \omega_{k}(\xi) \in T_{k}(\xi)$, the relations (66) (for $\alpha+e_{n}$ ), (67), (54) and the Hölder inequality

$$
\begin{aligned}
& |U(\xi)| \leqslant c_{1}(\alpha, \theta)\left|\gamma_{k}^{\prime}(\xi)-\omega_{k}(\xi)\right| l_{I^{(k)}}^{-n-|\alpha|-1} \quad \text { as } \omega_{(k)}(\xi) \neq 0, \\
& \left|\omega_{(k)} U\right| \leqslant c_{1}\left[\left|\omega-\gamma_{k+1}^{\prime}\right|+\left|\omega-\gamma_{k}^{\prime}\right|\right]\left|\omega-\gamma_{k}^{\prime}\right| l_{I^{(k)}}^{-n-|\alpha|-1} \text { in } \mathbb{R}^{n-1}, \\
& \left\|\omega_{(k)} U\right\|_{L^{1}\left(5 I^{(k)}\right)} \leqslant c(\alpha, \theta) l_{I^{(k)}}^{-|\alpha|} b_{I^{(k)}}^{2} .
\end{aligned}
$$

In the same way,

$$
\begin{aligned}
& |V(\xi, \tau)| \leqslant c(\alpha, \theta)\left|\omega_{(k)}(\xi)\right| l_{I^{(k)}}^{-n-|\alpha|-1} \quad \text { as } \omega_{(k)}(\xi) \neq 0 \text { and } 0 \leqslant \tau \leqslant 1 \\
& \left\|\omega_{(k)} \int_{0}^{1} V(\cdot, \tau) d \tau\right\|_{L^{1}\left(5 I^{(k)}\right)} \leqslant c(\alpha, \theta) l_{I^{(k)}}^{-|\alpha|} b_{I^{(k)}}^{2} .
\end{aligned}
$$


Hence, in view of the identity $\left.U\right|_{\mathbb{R}^{n-1} \backslash 5 I^{(k)}} \equiv 0$ we obtain

$$
\begin{aligned}
& \omega_{(k)}(\xi)\left\{U(\xi)+\int_{0}^{1} V(\xi, \tau) d \tau\right\}=\omega_{(k)}(\xi) D_{x}^{\alpha}\left|x-\left(\xi, \gamma_{k}^{\prime}(\xi)\right)\right|^{-n} \\
& +\int_{\mathbb{R}} \chi(\xi, t) D_{x}^{\alpha}|x-(\xi, t)|^{-n} d t \\
& D_{x}^{\alpha} M_{k}(x, \xi)+D_{x}^{\alpha} N_{\mathfrak{x}}(x, \xi)=\frac{\omega_{(k)}(\xi)}{2}\left\{U(\xi)+\int_{0}^{1} V(\xi, \tau) d \tau\right\} \\
& +\frac{\omega_{(k)}\left(\xi^{*}\right)}{2}\left\{U\left(\xi^{*}\right)+\int_{0}^{1} V\left(\xi^{*}, \tau\right) d \tau\right\} \\
& \left\|D_{x}^{\alpha} M_{k}(x, \cdot)+D_{x}^{\alpha} N_{\mathfrak{x}}(x, \cdot)\right\|_{L^{1}\left(\mathbb{R}^{n-1}\right)} \leqslant c(\alpha, \theta) l_{I^{(k)}}^{-|\alpha|} b_{I^{(k)}}^{2}+\frac{1}{2}\|\Theta\|_{L^{1}\left(\mathbb{R}^{n-1} \backslash 5 I^{(k)}\right)},
\end{aligned}
$$

where

$$
\Theta(\xi)=\omega_{(k)}(\xi) \int_{0}^{1} V(\xi, \tau) d \tau+\omega_{(k)}\left(\xi^{*}\right) \int_{0}^{1} V\left(\xi^{*}, \tau\right) d \tau .
$$

Let $\xi \in \mathbb{R}^{n-1} \backslash \overline{5 I^{(k)}}$. By 66 , 69 and $(70)$ we get

$$
\begin{aligned}
& \left|\int_{0}^{1} V(\xi, \tau) d \tau\right| \leqslant \frac{c(\alpha, \theta)\left|\omega_{(k)}(\xi)\right|}{|\mathfrak{x}-\xi|^{n+|\alpha|+1}} \leqslant c(\alpha, \theta) b_{I^{(k)}}|\mathfrak{x}-\xi|^{-n-|\alpha|}, \\
& |\Theta(\xi)| \leqslant \frac{c(\alpha, \theta) l_{I^{(k)}} b_{I^{(k)}}^{2}}{|\mathfrak{x}-\xi|^{n+|\alpha|}}+c(n)|\mathfrak{x}-\xi| b_{I^{(k)}}\left|\int_{0}^{1}\left[V\left(\xi^{*}, \tau\right)-V(\xi, \tau)\right] d \tau\right| .
\end{aligned}
$$

Hence, as $\alpha \neq 0$,

$$
\|\Theta\|_{L^{1}\left(\mathbb{R}^{n-1} \backslash 5 I^{(k)}\right)} \leqslant c(\alpha, \theta) l_{I^{(k)}}^{-|\alpha|} b_{I^{(k)}}^{2} .
$$

Let $\alpha=0$ and therefore,

$$
\begin{aligned}
& V(\xi, \tau)=\omega_{(k)}(\xi) \tau \int_{0}^{1} D_{x_{n}}\left|x-\left(\xi, \omega_{k}(\xi)+\omega_{(k)}(\xi) \tau \sigma\right)\right|^{-n} d \sigma, \\
& V\left(\xi^{*}, \tau\right)=\omega_{(k)}\left(\xi^{*}\right) \tau \int_{0}^{1} D_{x_{n}}\left|x-\left(\xi^{*}, \omega_{k}\left(\xi^{*}\right)+\omega_{(k)}\left(\xi^{*}\right) \tau \sigma\right)\right|^{-n} d \sigma .
\end{aligned}
$$

Now in view of (69), (70) and $(73)$

$$
\begin{aligned}
& \left|V(\xi, \tau)-V\left(\xi^{*}, \tau\right)\right| \leqslant \frac{c(n) l_{I^{(k)}} b_{I^{(k)}}}{|\mathfrak{x}-\xi|^{n+1}}+c(n)|\mathfrak{x}-\xi| b_{I^{(k)}} \max _{0 \leqslant \rho \leqslant 1}|\Theta(\xi, \rho)|, \\
& \Theta(\xi, \rho) \equiv D_{x_{n}}\left|x-\left(\xi, \omega_{k}(\xi)+\omega_{(k)}(\xi) \rho\right)\right|^{-n}+D_{x_{n}}\left|x-\left(\xi^{*}, \omega_{k}\left(\xi^{*}\right)+\omega_{(k)}\left(\xi^{*}\right) \rho\right)\right|^{-n} .
\end{aligned}
$$

We consider the center $X$ of the corresponding segment:

$$
2 X=\left(\xi, \omega_{k}(\xi)+\omega_{(k)}(\xi) \rho\right)+\left(\xi^{*}, \omega_{k}\left(\xi^{*}\right)+\omega_{(k)}\left(\xi^{*}\right) \rho\right) .
$$

By relations 46), $\left.\mathfrak{x}=\mathfrak{c}_{I^{(k)}}, \mathfrak{X}=\left(\mathfrak{x}, \gamma_{k}^{\prime}(\mathfrak{x})\right), \gamma_{k}^{\prime}=\gamma_{I^{(k)}}, 48\right), \omega_{k}(\xi)=\gamma_{k}^{\prime}(\xi), \omega_{k}\left(\xi^{*}\right)=\gamma_{k}^{\prime}\left(\xi^{*}\right)$, $\omega_{k+1}(\xi)=\gamma_{k+1}^{\prime}(\xi), \omega_{k+1}\left(\xi^{*}\right)=\gamma_{k+1}^{\prime}\left(\xi^{*}\right)$, 44) and (55) we obtain

$$
\begin{aligned}
& \left|x-g\left(\mathfrak{c}_{I^{(k)}}^{\square}\right)\right| \leqslant c(n, \theta)\left|\mathfrak{g}(x)-\mathfrak{c}_{I^{(k)}}^{\square}\right| \leqslant c(n, \theta) l_{I^{(k)}}, \\
& \left|g\left(\mathfrak{c}_{I^{(k)}}^{\square}\right)-\mathfrak{X}\right|=\left|w\left(\mathfrak{c}_{I^{(k)}}^{\square}\right)+3 W l_{I^{(k)}} / 2-\gamma_{k}^{\prime}(\mathfrak{x})\right|<2 W l_{I^{(k)}}, \\
& X=\mathfrak{X}+\left(0,\left(\gamma_{k+1}^{\prime}(\mathfrak{x})-\gamma_{k}^{\prime}(\mathfrak{x})\right) \rho\right), \quad|x-X| \leqslant c(n, \theta) l_{I^{(k)}} .
\end{aligned}
$$


Similarly to $(73)$ we have

$$
\begin{aligned}
& D_{X_{n}}\left|X-\left(\xi, \omega_{k}(\xi)+\omega_{(k)}(\xi) \rho\right)\right|^{-n}+D_{X_{n}}\left|X-\left(\xi^{*}, \omega_{k}\left(\xi^{*}\right)+\omega_{(k)}\left(\xi^{*}\right) \rho\right)\right|^{-n}=0, \\
& |\tau x+(1-\tau) X-(\xi, t)| \geqslant \frac{4}{5 \sqrt{n-1}}|\mathfrak{x}-\xi| \quad \text { for all } \tau \in[0,1] \text { and } t \in \mathbb{R}, \\
& |\Theta(\xi, \rho)| \leqslant c(n)|x-X||\mathfrak{x}-\xi|^{-n-2} \leqslant c(n, \theta) l_{I^{(k)}}|\mathfrak{x}-\xi|^{-n-2}, \\
& \left|V(\xi, \tau)-V\left(\xi^{*}, \tau\right)\right| \leqslant c(n, \theta) l_{I^{(k)}} b_{I^{(k)}}|\mathfrak{x}-\xi|^{-n-1}, \\
& |\Theta(\xi)| \leqslant c(n, \theta) l_{I^{(k)}} b_{I^{(k)}}^{2}|\mathfrak{x}-\xi|^{-n} .
\end{aligned}
$$

This implies (82) as $\alpha=0$.

By inequality 82 we get that

$$
\left\|D_{x}^{\alpha} M_{k}(x, \cdot)+D_{x}^{\alpha} N_{\mathfrak{x}}(x, \cdot)\right\|_{L^{1}\left(\mathbb{R}^{n-1}\right)} \leqslant c(\alpha, \theta) l_{I^{(k)}}^{-|\alpha|} b_{I^{(k)}}^{2} .
$$

We have $g\left(I^{\varpi}\right) \subset H_{0} \subset \Omega_{0}$ and $g\left(I^{\varpi}\right) \subset \bigcap_{j=1}^{\infty} \Omega_{j}$ (see $4 \mathrm{a}$ ) and hence, the condition $x \notin \operatorname{supp} \chi$ of Lemma 6 holds true. This is why

$$
\begin{aligned}
& S_{k+1}-S_{k}=\frac{\Gamma(n / 2)}{\pi^{n / 2}} s \quad \text { in the vicinity of each point } x \in g\left(I^{\square}\right), \\
& D^{\alpha}\left(F_{(k)}+S_{k+1}-S_{k}\right)(x)=\frac{\Gamma(n / 2)}{\pi^{n / 2}} \int_{\mathbb{R}^{n-1}}\left[D_{x}^{\alpha} M_{k}(x, \xi)+D_{x}^{\alpha} N_{\mathfrak{x}}(x, \xi)\right] d \xi
\end{aligned}
$$

due to the definition of the function $s(x)$ and identities (64b) and (75b). This leads us to (81).

By (44), 80) and (81) we conclude that

$$
\left\|D^{\alpha} S_{k+1}-D^{\alpha} S_{k}\right\|_{L^{\infty}\left(g\left(I^{\square}\right)\right)} \leqslant c(\alpha, \theta) l_{I^{(k)}}^{-|\alpha|} b_{I^{(k)}}, \quad \alpha \in \mathbb{N}_{0}^{n} .
$$

The series of the right hand sides converges thanks to $\Theta_{1}<\infty$, this is why the $\operatorname{limit}_{k \rightarrow \infty}\left(S_{k}-\right.$ $\left.S_{0}\right)$ exists in $C^{\infty}\left(g\left(I^{\square}\right)\right)$ and is obviously equal to $S-S_{0}$. This yields $(76)$. Differentiating the composition and applying (45), (46) and (76), we have

$$
\begin{aligned}
& \left\|D^{\alpha}\left(S \circ g-S_{0} \circ g\right)\right\|_{L^{\infty}\left(I^{\square}\right)} \leqslant c(\alpha, \theta) \sum_{k=0}^{\infty} l_{I^{(k)}}^{-1} b_{I^{(k)}}, \quad|\alpha|=1, \\
& \left\|D^{\alpha}\left(S \circ g-S_{0} \circ g\right)\right\|_{L^{\infty}\left(I^{\square}\right)} \leqslant c(\alpha, \theta) \sum_{j=0}^{1} l_{I}^{(1-|\alpha|) j} b_{I}^{j} \sum_{k=0}^{\infty} l_{I^{(k)}}^{(j-1)|\alpha|-j} b_{I^{(k)}}, \quad|\alpha| \geqslant 2 .
\end{aligned}
$$

In view of (2), (44) and (76), we obtain estimates (77) and (78).

By (60) and (63) we have

$$
F_{k}=W F_{(k)} \circ h^{k}
$$

on the cube $I^{\varpi}$, where

$$
h^{k}(x)=\left(x^{\prime}, \gamma_{k}^{\prime}\left(x^{\prime}\right)+W x_{n}\right), \quad x_{n}>0 .
$$

By (2), (43) and Lemma 5 we get the estimate

$$
\left\|\left(D^{\alpha} F_{(k)}\right) \circ h^{k}\right\|_{I} \leqslant c(\alpha, \theta) l_{I^{(k)}}^{-|\alpha|} b_{I^{(k)}}, \quad \alpha \in \mathbb{N}_{0}^{n} .
$$

For $x \in I^{\square}$, by (53) and (55),

$$
\begin{aligned}
\left|g(x)-h^{k}(x)\right| & =\left|g_{n}(x)-h_{n}^{k}(x)\right|=\left|w(x)-\gamma_{k}^{\prime}\left(x^{\prime}\right)\right| \\
& \leqslant\left|w(x)-\gamma_{0}^{\prime}\left(x^{\prime}\right)\right|+\left|\gamma_{0}^{\prime}\left(x^{\prime}\right)-\gamma_{k}^{\prime}\left(x^{\prime}\right)\right| \leqslant c(n) \sum_{j=0}^{k} l_{I^{(j)}} b_{I^{(j)}} .
\end{aligned}
$$


The points $g(x)$ and $h^{k}(x)$ belong to the convex set $H_{k}$ and hence, by Lemma 5 and inequality (81),

$$
\begin{aligned}
& \left|D^{\alpha} F_{(k)}\right|_{g(x)}-\left.D^{\alpha} F_{(k)}\right|_{h^{k}(x)}|\leqslant| g(x)-h^{k}(x)|\sup | D^{\alpha+e_{n}} F_{(k)} \mid \leqslant c(\alpha, \theta) l_{I^{(k)}}^{-|\alpha|} b_{I^{(k)}} \sum_{j=0}^{k} 2^{j-k} b_{I^{(j)}}, \\
& \left\|f_{k, \alpha}\right\|_{L^{\infty}\left(I^{\square}\right)} \leqslant c(\alpha, \theta) l_{I^{(k)}}^{-|\alpha|} b_{I^{(k)}} \sum_{j=0}^{k} 2^{j-k} b_{I^{(j)}},
\end{aligned}
$$

where

$$
f_{k, \alpha}=\left(D^{\alpha} F_{(k)}\right) \circ h^{k}+\left(D^{\alpha} S_{k+1}-D^{\alpha} S_{k}\right) \circ g .
$$

It is obvious that on the cube $I^{\square}$,

$$
D_{i} f_{k, \alpha}=\sum_{p=1}^{n}\left\{\left[\left(D^{\alpha+e_{p}} F_{(k)}\right) \circ h^{k}\right]\left[D_{i} h_{p}^{k}-D_{i} g_{p}\right]+f_{k, \alpha+e_{p}} D_{i} g_{p}\right\} .
$$

Hence, in view of (2), (46), (83) and (85), for each $\alpha \in \mathbb{N}_{0}^{n}$ we conclude that

$$
\begin{aligned}
& \left.\left\|D_{i} g_{p}-D_{i} h_{p}^{k}\right\|_{I} \leqslant c(n) \sum_{j=0}^{k} b_{I^{(j)}} \quad \text { (by analogy with }(84)\right), \\
& \left\|D_{i} g_{p}\right\|_{I} \leqslant\left\|D_{i} g_{p}\right\|_{L^{\infty}\left(I^{\square}\right)}+n l_{I}\left\|D\left(D_{i} g_{p}\right)\right\|_{L^{\infty}\left(I^{\square}\right)} \leqslant c(n, \theta), \\
& \left\|D_{i} f_{k, \alpha}\right\|_{L^{\infty}\left(I^{\square}\right)} \leqslant c(\alpha, \theta) l_{I^{(k)}}^{-|\alpha|-1} b_{I^{(k)}} \sum_{j=0}^{k} b_{I^{(j)}}, \\
& \left\|f_{k, \alpha}\right\|_{I} \leqslant c(\alpha, \theta) l_{I^{(k)}}^{-|\alpha|} b_{I^{(k)}} \sum_{j=0}^{k} 2^{j-k} b_{I^{(j)}}, \\
& \left\|D_{i} f_{k, \alpha}\right\|_{I} \leqslant c(\alpha, \theta) l_{I^{(k)}}^{-|\alpha|-1} b_{I^{(k)}} \sum_{j=0}^{k} b_{I^{(j)}} .
\end{aligned}
$$

Let us check the inequality

$$
\left\|f_{k, \alpha}^{\prime}\right\|_{I} \leqslant c(\alpha, \theta) l_{I^{(k)}}^{-|\alpha|} b_{I^{(k)}} \sum_{j=0}^{k} 2^{(j-k)(1-|\alpha|)} b_{I^{(j)}}, \quad|\alpha| \leqslant 2,
$$

for the function $f_{k, \alpha}^{\prime}=D^{\alpha} f_{k, 0}$. As $\alpha=0$, it is identical to $87 \mathrm{c}$, while as $|\alpha|=1$, it coincides with estimate $87 \mathrm{~d}$ ) for $\alpha=0$. Differentiating formula 86 , we get the identity

$$
\begin{aligned}
D_{i \jmath} f_{k, 0}= & \sum_{p=1}^{n}\left\{-\left[\left(D_{p} F_{(k)}\right) \circ h^{k}\right] D_{i \jmath} g_{p}+f_{k, e_{p}} D_{i \jmath} g_{p}+\left(D_{\jmath} f_{k, e_{p}}\right) D_{i} g_{p}\right\} \\
& +\sum_{p, q=1}^{n}\left[\left(D_{p q} F_{(k)}\right) \circ h^{k}\right]\left[D_{i} h_{p}^{k}-D_{i} g_{p}\right] D_{\jmath} h_{q}^{k} .
\end{aligned}
$$

Applying (83), (87) and the inequalities

$$
\left\|D_{i \jmath} g_{p}\right\|_{I} \leqslant c(n) l_{I}^{-1} b_{I}, \quad\left\|D_{\jmath} h_{q}^{k}\right\|_{I} \leqslant c(n, \theta), \quad \sum_{j=0}^{k} 2^{j-k} b_{I^{(j)}} \leqslant c(n, \theta)
$$

implied by (2) and (43)- 45), we arrive at estimate (88) with $|\alpha|=2$. 
For each $\delta \in \mathbb{R}$, by 88 and the Cauchy inequality we obtain

$$
\begin{aligned}
& \sum_{k=0}^{\infty}\left\|f_{k, \alpha}^{\prime}\right\|_{I} \leqslant c_{2}(\alpha, \theta) \Lambda^{1 / 2}\left(\sum_{k=0}^{\infty} l_{I^{(k)}}^{-\varepsilon-|\alpha|}\left(\sum_{j=0}^{k} 2^{(j-k)(1-|\alpha|)} b_{I^{(j)}}\right)^{2}\right)^{1 / 2}, \\
& \left(\sum_{j=0}^{k} 2^{(j-k)(1-|\alpha|)} b_{I^{(j)}}\right)^{2} \leqslant\left(\sum_{j=0}^{k} 2^{-2 j \varepsilon+2(j-k) \delta}\right) \sum_{j=0}^{k} 2^{2 j \varepsilon+2(j-k)(1-|\alpha|-\delta)} b_{I^{(j)}}^{2},
\end{aligned}
$$

where $\Lambda=\sum_{k=0}^{\infty} l_{I^{(k)}}^{\varepsilon-|\alpha|} b_{I^{(k)}}^{2}$. Let $\delta=\frac{1}{4}$ as $|\alpha| \leqslant 1$ and $\delta=0$ as $|\alpha|=2$, so that

$$
\begin{aligned}
& \sum_{j=0}^{k} 2^{-2 j \varepsilon+2(j-k) \delta} \leqslant c_{3}(\alpha, \varepsilon) \\
& \sum_{k=0}^{\infty}\left\|f_{k, \alpha}^{\prime}\right\|_{I} \leqslant c_{2} c_{3}^{1 / 2} l_{I}^{-\frac{\varepsilon+|\alpha|}{2}} \Lambda^{1 / 2}\left(\sum_{j=0}^{\infty} 2^{2 j(\varepsilon+1-|\alpha|-\delta)} b_{I^{(j)}}^{2} \sum_{k=j}^{\infty} 2^{k(-\varepsilon+|\alpha|-2+2 \delta)}\right)^{1 / 2} \\
& \leqslant c_{4}(\alpha, \theta, \varepsilon) l_{I}^{-\frac{\varepsilon+|\alpha|}{2}} \Lambda^{1 / 2}\left(\sum_{j=0}^{\infty} 2^{j(\varepsilon-|\alpha|)} b_{I^{(j)}}^{2}\right)^{1 / 2}=c_{4} l_{I}^{-\varepsilon} \Lambda
\end{aligned}
$$

But $\Lambda<\infty$ due to $\Theta_{2}<\infty$, and this is why the series

$$
\sum_{k=0}^{\infty} f_{k, 0}=\sum_{k=0}^{\infty}\left\{F_{(k)} \circ h^{k}+S_{k+1} \circ g-S_{k} \circ g\right\}
$$

converges absolutely in $C^{2, \mu}\left(I^{\varpi}\right)$. By the same convergence of the series $F=\sum_{k=0}^{\infty} W F_{(k)} \circ h^{k}$ (Theorem 22 and the aforementioned relation $C^{\infty}\left(g\left(I^{\square}\right)\right)-\lim _{k \rightarrow \infty}\left(S_{k}-S_{0}\right)=S-S_{0}$ we have the identity

$$
W \sum_{k=0}^{\infty} f_{k, 0}=F+W S \circ g-W S_{0} \circ g .
$$

Together with 89 it proves 79 ).

Let us calculate $S_{\Omega}$, when $\Omega$ is a half-space. We introduce the distance function

$$
\varrho_{\omega}(x)=\min _{\xi \in \mathbb{R}^{n-1}}|x-(\xi, \omega(\xi))|, \quad x \in \mathbb{R}^{n} .
$$

Theorem 4. If $\omega \in \mathbb{P}_{1}^{n-1}$, then

$$
\left.S \equiv \ln \varrho_{\omega}\right|_{\Omega}+\sigma_{n}
$$

where

$$
\sigma_{n}= \begin{cases}\ln 2+\sum_{k=1}^{\frac{n-2}{2}} \frac{1}{2 k}, & n \text { is even }, \\ \sum_{k=0}^{\frac{n-3}{2}} \frac{1}{2 k+1}, & n \text { is odd. }\end{cases}
$$

Proof. While checking (91), we can assume that $\Omega=\mathbb{R}_{+}^{n}$ and $x=\left(0, x_{n}\right)$. We introduce the spherical coordinates

$$
\begin{aligned}
& y_{1}=\rho \cos \phi_{2} \cos \phi_{3} \ldots \cos \phi_{n}, \\
& y_{2}=\rho \sin \phi_{2} \cos \phi_{3} \ldots \cos \phi_{n}, \\
& \ldots \\
& y_{n-1}=\rho \sin \phi_{n-1} \cos \phi_{n}, \\
& x_{n}-y_{n}=\rho \sin \phi_{n} .
\end{aligned}
$$


The set $\left\{y \in \mathbb{R}^{n}: y_{1} \ldots y_{n-1}\left(x_{n}-y_{n}\right) \neq 0\right\}$ is described as

$$
\rho>0, \quad\left|\phi_{2}\right| \in(0, \pi / 2) \cup(\pi / 2, \pi), \quad 0<\left|\phi_{3}\right|, \ldots,\left|\phi_{n}\right|<\pi / 2 .
$$

By the formula for the change of variables

$$
\Theta(r):=\int_{\substack{y \in \mathbb{R}^{n} \backslash \mathbb{R}_{+}^{n}: \\|x-y|<r}}|x-y|^{-n} d y=\int \frac{\cos \phi_{3} \cos ^{2} \phi_{4} \ldots \cos ^{n-2} \phi_{n}}{\rho} d \rho d \phi_{2} \ldots d \phi_{n},
$$

where the right integral is taken under the restrictions $x_{n} \leqslant \rho \sin \phi_{n}$ and $\rho<r$. We have

$$
\begin{aligned}
& \int_{(-\pi / 2, \pi / 2)^{n-2}} \cos \phi_{3} \ldots \cos ^{n-3} \phi_{n-1} d \phi_{2} \ldots d \phi_{n-1}=\frac{\operatorname{vol} \mathbb{S}^{n-2}}{2}=\frac{\pi^{\frac{n-1}{2}}}{\Gamma\left(\frac{n-1}{2}\right)}, \\
& \Theta(r)=\operatorname{vol} \mathbb{S}^{n-2} \int_{\rho>0 \&-\pi / 2<\phi<\pi / 2: x_{n} \leqslant \rho \sin \phi<r \sin \phi} \frac{\cos ^{n-2} \phi}{\rho} d \rho d \phi .
\end{aligned}
$$

The cases $n=2$ and $n>2$ should be studied independently. If $r>x_{n}$, then

$$
\begin{aligned}
\Theta(r)= & \operatorname{vol} \mathbb{S}^{n-2} \int_{\arcsin \frac{x_{n}}{r}}^{\pi / 2} \cos ^{n-2} \phi d \phi \int_{\frac{x_{n}}{\sin \phi}}^{r} \frac{d \rho}{\rho} \\
= & \operatorname{vol} \mathbb{S}^{n-2} \int_{\arcsin \frac{x_{n}}{r}}^{\pi / 2}\left(\ln \frac{r}{x_{n}}+\ln \sin \phi\right) \cos ^{n-2} \phi d \phi \\
= & \frac{\operatorname{vol} \mathbb{S}^{n-1}}{2} \ln \frac{r}{x_{n}}+O\left(\frac{x_{n}}{r} \ln \frac{r}{x_{n}}\right) \\
& +\operatorname{vol} \mathbb{S}^{n-2} \int_{0}^{\pi / 2}(\ln \sin \phi) \cos ^{n-2} \phi d \phi+O\left(\frac{x_{n}}{r} \ln \frac{r}{x_{n}}\right) \quad \text { as } r \rightarrow \infty,
\end{aligned}
$$

since

$$
\int_{-\pi / 2}^{\pi / 2} \cos ^{n-2} \phi d \phi=\operatorname{vol} \mathbb{S}^{n-1} / \operatorname{vol} \mathbb{S}^{n-2}
$$

This gives (91) with the constant

$$
\sigma_{n}=-\frac{2 \operatorname{vol} \mathbb{S}^{n-2}}{\operatorname{vol} \mathbb{S}^{n-1}} \int_{0}^{\pi / 2}(\ln \sin \phi) \cos ^{n-2} \phi d \phi .
$$

It is easy to see that $\sigma_{2}=\ln 2$ and $\sigma_{3}=1$. We integrate by parts:

$$
n \int_{0}^{\pi / 2}(\ln \sin \phi) \cos ^{n} \phi d \phi=(n-1) \int_{0}^{\pi / 2}(\ln \sin \phi) \cos ^{n-2} \phi d \phi-\int_{0}^{\pi / 2} \cos ^{n} \phi d \phi .
$$

Hence, $\sigma_{n+2}=\sigma_{n}+\frac{1}{n}$, which proves 92 .

The next theorem is the main result of the paper. Together with Theorems 1, 3, 4 and Lemma 7 it is aimed for proving (1) and related formulae.

Theorem 5. Given $\omega \in \operatorname{LIP}$ and $\theta \geqslant\|\omega\|_{\text {Lip }}$, let $\left(\left\{\gamma_{K}\right\}, w, W, g, \mathfrak{g}, \mathfrak{G}, A, \lambda, L\right)$ be a standard set of the pair $(\omega, \theta)$. Then $L \in \mathrm{VL}(0)$ and the potential

$$
\Phi_{L}(x)=\int_{y_{n}>0} E(A ; x, y) L(y) d y
$$

and the function $S \equiv S_{\Omega}$ satisfy the inequality

$$
\left\|D_{n} \Phi_{L}-\mathbf{x}_{n}^{-1} \Phi_{L}+1-\mathbf{x}_{n} D_{n}(S \circ g)\right\|_{I} \leqslant c(n, \theta, \mu) \sum_{J} \Gamma_{I J}^{(1, n)} b_{J}^{2}
$$


for each $I \in \mathcal{D}$. The function $\varrho_{\gamma_{I}} \circ g$ is positive on $I^{\square}$ (see (90) and the estimate

$$
\left\|D_{i j}\left\{\Phi_{L}-W^{-1} w+\mathbf{x}_{n}\left[\ln \varrho_{\gamma_{I}} \circ g-S \circ g\right]\right\}\right\|_{I} \leqslant \frac{c(n, \theta, \mu)}{l_{I}} \sum_{J} \Gamma_{I J}^{(1, n)} b_{J}^{2}
$$

holds true.

Proof. Lemma 4 allows us to apply Theorem 2 to the function $f=w$ and to apply Theorem 7. In this way, the notations $f, \Theta, \overleftarrow{I J}, \overrightarrow{I J}, \mathcal{F}_{J}, \mathfrak{c}_{k}, A_{k}, \gamma_{k}, w_{k}, w_{(k)}, F_{k}, F, \gamma^{\prime}, \mathcal{R}_{w}, \Psi, \Theta^{*}, \Theta_{1}, \Theta_{2}$, $\Theta_{2}^{*}, \gamma_{k}^{\prime}, \tau_{i, k}, \omega_{(k)}, \omega_{k}, \varphi_{k}^{\prime}, \tau_{s, \infty}, \Omega_{k}$ and $S_{k}$ make sense. The belonging $L \in \operatorname{VL}(0)$ is implied by (52) and the relation $\Theta_{1}<\infty$. The integral $\Phi_{L}(x)$ is well-defined by Theorem 1 . The function $\varrho_{\gamma_{I}} \circ g$ is positive on $I^{\triangleright}$ due to the identity $\gamma_{I}=\gamma_{0}^{\prime}$ and the belonging $g\left(I^{\square}\right) \subset H_{0}$ (see the statement after (63)).

The function $U(x)=x_{n}$ is harmonic in the domain $\Omega$. Therefore, by the remark after Theorem 3 and by inequalities (2), (45) and (52) we have

$$
\begin{aligned}
& A w=A g_{n}=L D_{n} g_{n}=L D_{n} w+W L, \\
& \|A w-W L\|_{J} \leqslant\left\|D_{n} w\right\|_{J}\|L\|_{J} \leqslant c(n, \theta) l_{J}^{-1} b_{J}^{2}, \quad J \in \mathcal{D} .
\end{aligned}
$$

By inequality (9) in Theorem 1 we obtain

$$
\begin{aligned}
& \left\|W D_{n} \Phi_{L}-W \mathbf{x}_{n}^{-1} \Phi_{L}-D_{n} \Phi_{A w}+\mathbf{x}_{n}^{-1} \Phi_{A w}\right\|_{I} \leqslant c(n, \theta, \mu) \Theta_{2}^{*}, \\
& \left\|W D_{i j} \Phi_{L}-D_{i j} \Phi_{A w}\right\|_{I} \leqslant c(n, \theta, \mu) l_{I}^{-1} \Theta_{2}^{*} .
\end{aligned}
$$

At the same time,

$$
\begin{aligned}
& \left\|D_{n} \Phi_{A w}-\mathbf{x}_{n}^{-1} \Phi_{A w}-D_{n} \Psi+\mathbf{x}_{n}^{-1} \Psi\right\|_{I} \leqslant c(n, \theta, \mu) \Theta_{2}^{*}, \\
& \left\|D_{i j} \Phi_{A w}-D_{i j} \Psi\right\|_{I} \leqslant c(n, \theta, \mu) l_{I}^{-1} \Theta_{2}^{*}
\end{aligned}
$$

due to estimates (26b) and (57) in Theorem 2 and to Lemma 4. By (59),

$$
D_{n} \Psi-\mathbf{x}_{n}^{-1} \Psi=D_{n} w-\mathbf{x}_{n}^{-1} w+\mathbf{x}_{n}^{-1} \gamma_{0}-\mathbf{x}_{n} D_{n} F, \quad D_{i j} \Psi=D_{i j} w-D_{i j}\left(\mathbf{x}_{n} F\right) .
$$

By inequality (79) in Lemma 7 with $\varepsilon=|\alpha|-1 \in\{0,1\}$ we obtain

$$
\begin{aligned}
& \left\|\mathbf{x}_{n} D_{n} F+\mathbf{x}_{n} D_{n}\left[W S \circ g-W S_{0} \circ g\right]\right\|_{I} \leqslant c(n, \theta) \Theta_{2}^{*}, \\
& \left\|D_{i j}\left(\mathbf{x}_{n} F\right)+D_{i j}\left(\mathbf{x}_{n}\left[W S \circ g-W S_{0} \circ g\right]\right)\right\|_{I} \leqslant c(n, \theta) l_{I}^{-1} \Theta_{2}^{*} .
\end{aligned}
$$

At that, $S_{0} \circ g=\ln \varrho_{\gamma_{I}} \circ g+\sigma_{n}$ on $I^{\square}$ by Theorem 4. The identity

$$
\begin{aligned}
& \left\{W D_{n} \Phi_{L}-W \frac{\Phi_{L}}{\mathbf{x}_{n}}-D_{n} \Phi_{A w}+\frac{\Phi_{A w}}{\mathbf{x}_{n}}\right\}+\left\{D_{n} \Phi_{A w}-\frac{\Phi_{A w}}{\mathbf{x}_{n}}-D_{n} \Psi+\frac{\Psi}{\mathbf{x}_{n}}\right\} \\
& +D_{n} w-\mathbf{x}_{n}^{-1} w+\mathbf{x}_{n}^{-1} \gamma_{0}-\left\{\mathbf{x}_{n} D_{n} F+\mathbf{x}_{n} D_{n}\left[W S \circ g-W S_{0} \circ g\right]\right\} \\
& =W\left[D_{n} \Phi_{L}-\mathbf{x}_{n}^{-1} \Phi_{L}+\mathbf{x}_{n} D_{n}\left[\ln \varrho_{\gamma_{I}} \circ g-S \circ g\right]\right]
\end{aligned}
$$

and the identity

$$
\begin{aligned}
\left\{W D_{i j} \Phi_{L}\right. & \left.-D_{i j} \Phi_{A w}\right\}+\left\{D_{i j} \Phi_{A w}-D_{i j} \Psi\right\}+D_{i j} w \\
& -\left\{D_{i j}\left(\mathbf{x}_{n} F\right)+D_{i j}\left(\mathbf{x}_{n}\left[W S \circ g-W S_{0} \circ g\right]\right)\right\}=W D_{i j}\left[\Phi_{L}+\mathbf{x}_{n}\left[\ln \varrho_{\gamma_{I}} \circ g-S \circ g\right]\right]
\end{aligned}
$$

show that checking inequalities (93) is reduced to checking the estimate

$$
\|u\|_{I} \leqslant c(n, \theta, \mu) \Theta_{2}^{*}
$$

where

$$
u=D_{n} w-\mathbf{x}_{n}^{-1} w+\mathbf{x}_{n}^{-1} \gamma_{0}+W-W \mathbf{x}_{n} D_{n}\left[\ln \varrho_{\gamma_{I}} \circ g\right]
$$


In view of the formula $\gamma_{I} \circ \mathbf{x}^{\prime}=\gamma_{0}$ we write

$$
\begin{aligned}
& \varrho_{\gamma_{I}} \circ g=C\left[g_{n}-\gamma_{I} \circ \mathbf{x}^{\prime}\right]=C\left[w+W \mathbf{x}_{n}-\gamma_{0}\right], \quad C=C\left(\nabla \gamma_{I}\right)>0, \\
& W \mathbf{x}_{n} D_{n}\left[\ln \varrho_{\gamma_{I}} \circ g\right]=W \mathbf{x}_{n} \frac{D_{n} w+W}{w+W \mathbf{x}_{n}-\gamma_{0}}=\frac{D_{n} w+W}{1+\frac{w-\gamma_{0}}{W \mathbf{x}_{n}}}, \\
& u=\frac{\gamma_{0}-w}{\mathbf{x}_{n}}+D_{n} w+W-\frac{D_{n} w+W}{1+\frac{w-\gamma_{0}}{W \mathbf{x}_{n}}}=\frac{\gamma_{0}-w}{\mathbf{x}_{n}} \frac{\frac{w-\gamma_{0}}{\mathbf{x}_{n}}-D_{n} w}{1+\frac{w-\gamma_{0}}{W \mathbf{x}_{n}}} W^{-1} .
\end{aligned}
$$

Due to (2), the Taylor formula, (45), 48) and (44) we have

$$
\begin{aligned}
& \left\|\frac{\gamma_{0}-w}{\mathbf{x}_{n}}\right\|_{I}=\left\|\frac{w-\gamma_{0}}{\mathbf{x}_{n}}\right\|_{I} \leqslant\left\|w-\gamma_{0}\right\|_{I}\left\|\mathbf{x}_{n}^{-1}\right\|_{I} \leqslant c_{1}(n) b_{I}, \\
& \left\|D_{n} w\right\|_{I} \leqslant c_{2}(n) b_{I}, \quad\left\|\frac{w-\gamma_{0}}{W \mathbf{x}_{n}}\right\|_{L^{\infty}\left(I^{\varpi}\right)} \leqslant \frac{1}{3}, \\
& \left\|\frac{1}{1+\frac{w-\gamma_{0}}{W \mathbf{x}_{n}}}\right\|_{I} \leqslant \frac{3}{2}+l_{I}^{\mu} \frac{9}{4}\left|\frac{w-\gamma_{0}}{W \mathbf{x}_{n}}\right|_{C^{\mu}\left(I^{\square}\right)} \leqslant \frac{3}{2}+\frac{9 c_{1} b_{I}}{4 W} \leqslant c_{3}(n, \theta), \\
& \|u\|_{I} \leqslant\left[c_{1} b_{I}\right]\left[c_{1} b_{I}+c_{2} b_{I}\right] c_{3} W^{-1} \leqslant c(n, \theta) \Theta_{2}^{*} .
\end{aligned}
$$

This completes the proof.

\section{BIBLIOGRAPHY}

1. A.I. Parfenov. Discrete Hölder estimates for a parametrix variation // Matem. Trudy. 17:1, 175201 (2014). [Siber. Adv. Math. 25:3, 209-229 (2015).]

2. R.A. Hunt, R.L. Wheeden. Positive harmonic functions on Lipschitz domains // Trans. Amer. Math. Soc. 147:2, 507-527 (1970).

3. D.S. Jerison, C.E. Kenig. Boundary behavior of harmonic functions in non-tangentially accessible domains // Adv. Math. 46:1, 80-147 (1982).

4. S.E. Warschawski. On conformal mapping of infinite strips // Trans. Amer. Math. Soc. 51:2, 280-335 (1942).

5. V. Kozlov, V. Maz'ya. Asymptotic formula for solutions to elliptic equations near the Lipschitz boundary // Ann. Mat. Pura Appl. (4). 184:2, 185-213 (2005).

6. V. Kozlov. Asymptotic representation of solutions to the Dirichlet problem for elliptic systems with discontinuous coefficients near the boundary // Electron. J. Diff. Equ. 2006:10, 1-46 (2006).

7. V. Kozlov. Behavior of solutions to the Dirichlet problem for elliptic systems in convex domains // Comm. Partial Diff. Equ. 34:1, 24-51 (2009).

8. K. Ramachandran. Asymptotic behavior of positive harmonic functions in certain unbounded domains // Potential Anal. 41:2, 383-405 (2014).

9. A.I. Parfenov. Weighted a priori estimate in straightenable domains of local Lyapunov-Dini type // Sibir. Electr. Matem. Izv. 9, 65-150 (2012). (in Russian).

10. A.I. Parfenov. A criterion for straightening a Lipschitz surface in the Lizorkin-Triebel sense. III // Matem. Trudy. 13:2, 139-178 (2010). [Siber. Adv. Math. 21:2, 100-129 (2011).]

Anton Igorevich Parfenov,

Sobolev Institute of Mathematics, SB RAS,

Akademik Koptyug av. 4,

630090, Novosibirsk, Russia

E-mail: parfenov@math.nsc.ru 